\title{
Temozolomide: The evidence for its therapeutic efficacy in malignant astrocytomas
}

This article was published in the following Dove Press journal:

Core Evidence

25 June 2009

Number of times this article has been viewed

\section{Ayman I Omar' \\ Warren P Mason ${ }^{2}$ \\ 'Department of Medicine, Princess Margaret Hospital and the University of Toronto, Toronto, Ontario, Canada; ${ }^{2}$ Department of Medicine, University of Toronto, Toronto, Ontario, Canada}

Introduction: Malignant gliomas are a heterogeneous group of primary central nervous system neoplasms that represent less than $2 \%$ of all cancers yet carry a significant burden to society. They are frequently associated with considerable and progressive neurological disability and are ultimately intractable to all forms of treatment. Temozolomide (TMZ) is a new second generation DNA alkylating agent that has become part of malignant astrocytoma management paradigms because of its proven efficacy, ease of administration, and favorable toxicity profile.

Aims: To review the role of TMZ in the management of malignant astrocytomas (World Health Organization grades III and IV) including newly diagnosed (n) and recurrent (r) anaplastic astrocytomas (AA) and glioblastomas.

Evidence review: A series of pivotal clinical trials have established a role for TMZ in the treatment of malignant astrocytomas. A large phase II trial examining the role of TMZ in rAA showed a response rate of $35 \%$, and a 6-month progression-free survival of $46 \%$. This led to the accelerated approval of TMZ by the FDA and the EU for the treatment of rAA. Evidence for a role of TMZ in nAA is currently limited but research is ongoing in this area. The role of TMZ in the management of glioblastoma at the time of recurrence (rGBM) is less impressive but evidence for its activity was demonstrated in two large phase II trials that led to the approval of TMZ for this indication in Europe and Canada but not in the US. A recent large prospective randomized phase III trial showed that the addition of TMZ during and after radiation therapy (RT) in newly diagnosed (nGBM) patients prolonged median overall survival by 2.5 months; perhaps more importantly, the 2-year survival rate for patients receiving TMZ and RT was 26\% compared with $10 \%$ for those receiving RT alone. Concurrent TMZ with RT followed by adjuvant TMZ has become the standard of care for nGBM patients. Based on the evidence presented in this trial, TMZ received approval from the FDA and the EU for patients with nGBM in 2005. Place in therapy: There is evidence to support the use of TMZ for the following diseases in the order of most to least convincing: nGBM, rAA, rGBM, and nAA. This order may quickly change as more trials are being designed and implemented, particularly with novel TMZ dosing schedules.

Keywords: temozolomide, evidence, malignant astrocytoma, glioma, anaplastic astrocytoma, glioblastoma

\section{Scope, aims, and objectives}

In the US, temozolomide (TMZ; Temodar ${ }^{\circledR}$, Temodal ${ }^{\circledR}$, Schering-Plough Corporation) is indicated for recurrent anaplastic astrocytoma (rAA) and newly diagnosed glioblastoma (nGBM), and in Europe for nGBM, recurrent glioglastoma (rGBM), and rAA. This review summarizes the disease background, current therapy options, and unmet medical needs for patients with malignant gliomas, and outlines the current evidence for the role 


\section{Core evidence place in therapy summary for temozolomide (TMZ) in malignant astrocytomas}

\begin{tabular}{|c|c|c|}
\hline Outcome measure & Evidence & Implications \\
\hline \multicolumn{3}{|l|}{ Patient-oriented evidence } \\
\hline $\begin{array}{l}\text { The addition of TMZ to standard RT followed by } \\
\text { adjuvant TMZ increases the percentage of patients } \\
\text { with PFS at } 6 \text { months ( } 53.9 \% \text { for TMZ/RT + TMZ vs } \\
36.4 \% \text { for RT alone, a } 17.2 \% \text { increase) }\end{array}$ & Clear & $\begin{array}{l}\text { TMZ increases the percentage of progression-free } \\
\text { nGBM patients }\end{array}$ \\
\hline $\begin{array}{l}\text { Concurrent TMZ/RT followed by adjuvant TMZ } \\
\text { increases mOS by } 2.5 \text { months compared with RT } \\
\text { alone in nGBM patients }\end{array}$ & Clear & $\begin{array}{l}\text { TMZ results in improved survival among nGBM } \\
\text { patients }\end{array}$ \\
\hline $\begin{array}{l}\text { Concurrent TMZ/RT followed by adjuvant TMZ } \\
\text { increases } 2 \text {-year survival from } 10 \% \text { to } 24 \% \text { for } \\
\text { nGBM patients treated with RT alone }\end{array}$ & Clear & $\begin{array}{l}\text { TMZ increases the odds of } n G B M \text { patients surviving } \\
\text { to the } 2 \text {-year time point }\end{array}$ \\
\hline $\begin{array}{l}\text { Concurrent TMZ/RT followed by adjuvant TMZ } \\
\text { results in a } 37 \% \text { reduction in the risk of death for } \\
\text { nGBM patients compared with RT alone }\end{array}$ & Clear & TMZ reduces the risk of death among $\mathrm{nGBM}$ patients \\
\hline $\begin{array}{l}\text { The HRQoL scores among nGBM patients treated } \\
\text { with concurrent TMZ/RT followed by adjuvant TMZ } \\
\text { were not inferior to the HRQoL scores derived } \\
\text { from patients treated with RT alone }\end{array}$ & $\begin{array}{l}\text { were not inferior to the HRQoL scores derived } \\
\text { from patients treated with RT alone }\end{array}$ & $\begin{array}{l}\text { TMZ does not reduce the quality of life among } n G B M \\
\text { patients }\end{array}$ \\
\hline $\begin{array}{l}\text { The PFS6m for rGBM patients treated with TMZ } \\
\text { was } 21 \% \text { compared with a PFS } 6 \mathrm{~m} \text { of only } 8 \% \text { for } \\
\text { patients treated with PCB, a } 13 \% \text { increase }\end{array}$ & Clear & $\begin{array}{l}\text { TMZ improves the percentage of progression-free } \\
\text { rGBM patients at } 6 \text { months compared with } P C B\end{array}$ \\
\hline $\begin{array}{l}\text { TMZ does not seem to improve survival among } \\
\text { rGBM or rAA patients }\end{array}$ & Limited & $\begin{array}{l}\text { TMZ does not improve survival when used for } \\
\text { recurrent disease }\end{array}$ \\
\hline $\begin{array}{l}\text { TMZ treatment results in improved HRQoL scores } \\
\text { when used for both rGBM and rAA patients }\end{array}$ & Clear & $\begin{array}{l}\text { TMZ improves patient quality of life when used for } \\
\text { recurrent disease }\end{array}$ \\
\hline \multicolumn{3}{|l|}{ Disease-oriented evidence } \\
\hline $\begin{array}{l}\text { nGBM patients treated with neoadjuvant TMZ } \\
\text { achieve an ORR of } 20 \%\end{array}$ & Moderate & $\begin{array}{l}\text { TMZ given prior to RT for } n G B M \text { patients results in } \\
\text { radiographic improvement that usually correlates with } \\
\text { clinical improvement }\end{array}$ \\
\hline $\begin{array}{l}\text { rGBM patients treated with TMZ did not achieve an } \\
\text { improved ORR compared with other agents }\end{array}$ & Limited & $\begin{array}{l}\text { TMZ given to rGBM patients does not result in a } \\
\text { better radiographic response compared to other } \\
\text { agents }\end{array}$ \\
\hline $\begin{array}{l}\text { TMZ possibly results in a higher ORR for rAA } \\
\text { patients ( } 35 \%) \text { compared with only a I } 4 \% \text { ORR } \\
\text { for patients treated with other chemotherapeutic } \\
\text { agents. Because of the differences in baseline patient } \\
\text { characteristics for those two trials, no definitive } \\
\text { conclusions can be drawn }\end{array}$ & Limited & $\begin{array}{l}\text { TMZ may result in a more significant radiographic } \\
\text { improvement when given to rAA patients compared } \\
\text { with other agents }\end{array}$ \\
\hline \multicolumn{3}{|l|}{ Economic evidence } \\
\hline $\begin{array}{l}\text { TMZ is an expensive new agent but the added costs } \\
\text { may be justified by the improved survival among } \\
\text { nGBM patients }\end{array}$ & Limited & $\begin{array}{l}\text { Despite the lack of conclusive cost-effectiveness data, } \\
\text { TMZ should be considered especially from nGBM } \\
\text { patients }\end{array}$ \\
\hline $\begin{array}{l}\text { The cost effectiveness of TMZ in recurrent disease } \\
\text { is not clear at this point }\end{array}$ & Limited & $\begin{array}{l}\text { One study concludes that TMZ may not be cost } \\
\text { effective in recurrent disease compared with } \\
\text { lomustine }\end{array}$ \\
\hline \multicolumn{3}{|c|}{$\begin{array}{l}\text { Abbreviations: } \mathrm{HRQ} \text { LL, health-related quality of life; } \mathrm{mOS} \text {, median overall survival; nGBM, newly diagnose survival; glioblastoma; ORR, objective radiographic response; } \\
\text { PCB, procarbazine; PFS, progression-free survival; PFS6m, progression-free survival at } 6 \text { months; rAA, recurrent anaplastic astrocytoma; rGBM, recurrent glioblastoma; } \\
\text { rGBM, recurrent glioblastoma; RT, radiotherapy. }\end{array}$} \\
\hline
\end{tabular}

of TMZ in the management of malignant gliomas. Outcomes evaluated included objective radiographic response, progression-free survival at 6 months (PFS6m), median overall survival (mOS), 2-year survival, health-related quality of life (HRQoL), and the safety/tolerability profile of TMZ when used for the above indications.

\section{Methods}

The English language medical literature was searched in the following databases.

- Medline

- EMBase

- Cochrane reviews 
The search terms used were temozolomide, glioblastoma, and anaplastic astrocytoma, and the records were limited to clinical trials, meta analyses, practice guidelines, and randomized controlled trials. The cutoff dates were from beginning through to March 25, 2008 (Table 1).

\section{Disease overview Epidemiology}

According to the Central Brain Tumor Registry of the US (CBTRUS) database, the number of reported cases of glioblastoma (GBM) between 1998-2002 was $12943 .{ }^{1}$ The age-adjusted incidence of GBM was 3.05 [confidence interval (CI): 3.00, 3.10] and the median age at diagnosis was 64 years (CBTRUS 2006). The number of reported anaplastic astrocytoma (AA) cases during the same period was 2029 and the age-adjusted incidence was 0.47 (CI: $0.45,0.49)$, with a median age at diagnosis for AA of 51 years. ${ }^{1}$

In the registry, GBM was the most common subtype of glioma and represented $20.3 \%$ of all malignant and nonmalignant brain tumors, and $50.7 \%$ of all glioma subtypes. In contrast, AA represented only $3.2 \%$ of all malignant and nonmalignant brain tumors, and $7.9 \%$ of all gliomas. The age-adjusted incidence rates for both GBM and AA were higher among males compared with females (3.86 per 100000 person years in males versus 2.39 per 100000 person years in females for GBM, and 0.56 per 100000 person years among males versus 0.38 per 100000 person years among females for AA).

Table I Evidence base included in the review ${ }^{\mathrm{a}}$

\begin{tabular}{ll}
\hline Category & Number of records \\
\cline { 2 - 2 } & Full papers \\
\hline Initial search & 96 \\
Records included & 58 \\
Records excluded & 38 \\
Search update & \\
Hand search of cross-references & 29 \\
Preclinical evidence & 9 \\
Level I evidence (systematic & 2 \\
review, meta analysis) & \\
Level 2 clinical evidence (RCT) & 9 \\
Level $\geq 3$ clinical evidence & 45 \\
Trials other than RCT & 42 \\
Case reports & 5 \\
Economic evidence & 10 \\
\hline
\end{tabular}

Notes: aSome references fall under multiple categories. For definition of levels of evidence, see the Core Evidence website (http://www. dovepress.com/core-evidence-journal).

Abbrevation: RCT, randomized controlled trial.
The incidence of both AA and GBM among white people was almost double the rate among black people. ${ }^{1}$

\section{Risk factors and etiology}

Familial, environmental, and genetic factors have been implicated in the development of astrocytomas. Most reported studies lack statistical power to identify unequivocally any single risk factor for the development of malignant gliomas.

\section{Familial factors}

Malignant astrocytomas are occasionally found at a higher frequency in families where several other malignancies are prevalent. Such familial cancer syndromes are due to germline mutations and include Li-Fraumeni syndrome, neurofibromatosis, Turcot syndrome, and multiple enchondromatosis. ${ }^{2}$ Interestingly, neurofibromatosis type- 1 is due to a mutation on chromosome 17 , which also harbors the p53 gene implicated in the genesis of many malignant gliomas. ${ }^{3}$

\section{Environmental factors}

Environmental factors that contribute to the development of malignant gliomas are notoriously difficult to ascertain with confidence. Because of the relatively low incidence of malignant gliomas, studies are able to establish a possible but inconclusive relationship between environmental exposures and the development of malignant gliomas. Ionizing radiation, however, has unequivocally been implicated in the genesis of secondary brain malignancies including AA and GBM after a minimallatency period of approximately 10 years. ${ }^{4,5}$ For instance, the routine use of prophylactic irradiation for leukemia has resulted in an increased risk of secondary gliomas in this patient population. ${ }^{6}$

On the other hand, there has been considerable debate as to whether high-energy electromagnetic radiation generated by the use of cellular phones contributes to the genesis of malignant gliomas. Results are conflicting but several studies have concluded that cellular telephones do not seem to be related causally to the development of malignant gliomas. ${ }^{8}$ Long-term follow up studies may still be needed however to settle this debate.

\section{Genetic mutations}

Gliomas are believed to arise as a result of a series of genetic aberrations that slowly accumulate over time. ${ }^{9}$ Theoretically, asthenumber of geneticmutationsincreases, tumors may progress from low-grade to higher grade phenotypes and genotypes. Molecular profiling has allowed the characterization of 
genetic mutations within brain tumor samples. GBMs can arise as a result of progression from low-grade astrocytomas, also known as secondary GBM, or de novo, also known as primary GBM. Primary (de novo) GBM represents approximately $80 \%$ of all GBMs. The genetic mutations within each of those entities may differ markedly. For example, mutation of the proapoptotic p53 gene located on chromosome 17 is believed to be the primary event involved in the genesis of a low-grade astrocytoma and is found with a higher incidence among secondary GBM tumor samples. ${ }^{3}$ Consequently, the mutated p53 gene is found with increasing frequency $(>65 \%)$ in diffuse [World Health Organization (WHO) grade II] astrocytomas. ${ }^{10}$ This molecular derangement typically results in defective apoptosis and allows for uncontrolled cellular proliferation. ${ }^{11}$ In addition, overexpression of the plateletderived growth factor receptor is found in over $60 \%$ of lowgrade astrocytomas. ${ }^{11}$ The further sequential accumulation of genetic mutations in the form of loss of heterozygosity for chromosome $10 \mathrm{q}$ ( $\mathrm{LOH} \mathrm{10q)}$, as well as mutation of the tumor suppressor retinoblastoma gene, has been observed in the progression of low-grade diffuse astrocytomas (WHO grade II) to AA (WHO grade III). ${ }^{12}$ Several genetic mutations are found in both GBM and AA or GBM arising from AA, but with variable frequency. For example, p53 mutation is far less frequent in primary GBM $(\sim 25 \%)$ when compared with AA $(\sim 50 \%)$ and secondary GBM $(\sim 65 \%) .{ }^{13}$ Additionally, LOH $10 \mathrm{q}$ is found in only $\sim 8 \%$ of GBM that have evolved from AA, compared with $\sim 50 \%$ of primary GBM. ${ }^{14}$ The amplification of the epidermal growth factor receptor seems more important in primary GBM $(\sim 36 \%)$ than in GBM that arises from an underlying AA $(\sim 8 \%) .{ }^{11}$ These differences may aid in the diagnosis and prognosis of AA and GBM, and potentially identify targeted therapies to specific tumor subtypes based on genetic features.

\section{Histologic subtypes}

Astrocytomas are classified in ascending order of aggressiveness into WHO grades I-IV. ${ }^{15}$ Malignant astrocytomas fall under the more aggressive WHO grades III and IV and include AA (WHO grade III) and the most aggressive astrocytic neoplasm, GBM, which is classified as a grade IV tumor in the WHO grading scheme (Figure 1). GBM is also the most frequently encountered primary brain malignancy in the adult population. ${ }^{11,16}$

\section{Goals of therapy}

The goals of surgery for nGBM and nAA differ from those for recurrent disease. The goals of surgery for nGBM and

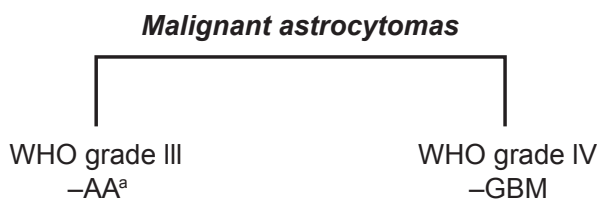

Figure I WHO classification scheme for malignant astrocytomas. Notes: a'SomeAAs containing oligodendroglial components are classified as anaplastic oligoastrocytomas (AOA).

Abbreviations: AA, anaplastic astrocytoma; GBM, glioblastoma multiforme; WHO, World Health Organization.

nAA include providing tissue for an accurate pathologic diagnosis as well as tumor cytoreducation that can alleviate symptoms and reduce neurological deficits. For recurrent disease, the goal is usually tumor debulking alone. Whenever surgical resection is possible, the goal is maximal feasible tumor reduction provided that this can be achieved safely. Postoperative radiotherapy (RT) and chemotherapy are given to treat any residual disease and achieve a durable remission.

Despite multimodality treatment, patients with malignant gliomas invariably relapse, and at this time treatment goals are palliative in nature to the extent that therapeutic efforts are focused on delaying further tumor progression and preserving quality of life.

\section{Evaluating response to therapy}

In the earlier phase I and II trials evaluating the safety and efficacy of TMZ, an objective radiographic response was often used as a primary endpoint. ${ }^{17,18}$ Recently, PFS6m has been utilized as a new primary outcome measure in neurooncology because objective radiographic responses are uncommon in trials of new agents for recurrent malignant glioma, and because disease stabilization (radiographic or clinical) has been perceived as a valuable and treatmentspecific outcome. The use of mOS and HRQoL have also been implemented as useful and informative secondary outcomes. To this extent, quality-adjusted life-year (QALY) is an emerging indicator incorporating both quantity and quality of life as a single parameter. ${ }^{19,20}$ In economic analyses, the cost of QALYs gained by administering a drug is an important measure of the cost effectiveness of the drug and may play an important role in drug reimbursement decisions. QALY is calculated by the duration spent in a health state (in years) weighted by the preference to that state (utility).

The following discussion defines the parameters employed as endpoints in the trials evaluating TMZ activity in malignant astrocytomas. 


\section{Objective radiographic response}

One of the most widely adopted measures of tumor response is the objective radiographic response (ORR) according to the criteria proposed by MacDonald et al. ${ }^{21}$ Imaging modalities include contrasted computerized tomography (CT) scans of the brain or gadolinium-enhanced magnetic resonance (MR) scans, and these scans are obtained at various time points following treatment. In this scale, the two largest perpendicular diameters of the enhancing mass are multiplied and the product is then used as a baseline measure of the "tumor size." Four response categories are identified on contrastenhanced CT or MR scans.

- Partial response (PR): a 50\% decrease in tumor size compared to a prior scan at least 1 month apart, on a stable ( $>2$ weeks) dose of steroids, and neurologically stable or improving.

- Complete response (CR): the complete disappearance of the contrast-enhancing mass.

- Progressive disease: an increase in tumor size $>25 \%$ compared to a prior scan at least 1 month apart, on a stable ( $>2$ weeks) dose of steroids, and neurologic worsening.

- Stable disease: all other response categories.

Some of the drawbacks associated with this technique include a relatively wide interobserver variability of the measurements (hence the importance of a central radiology review of all scans assessed in a clinical trial), the occasional presence of multifocal disease that can be difficult to measure, variations associated with scanning techniques (such as dose of contrast medium), and variations in the timing of contrast injections relative to image acquisition. Furthermore, since tumors are three-dimensional masses, a bidimensional quantitative measure often fails to reflect the true size and dimensions of the tumor. In addition, some gliomas enhance heterogeneously and may contain nonenhancing areas that may not be included in the measurements, while conversely some contrast-enhancing areas may not represent tumor, but rather postsurgical changes or radiation effect. Despite these limitations, the ORR is a commonly employed endpoint used to assess efficacy of chemotherapeutic agents for the treatment of malignant astrocytomas.

\section{Progression-free survival PFS6m}

PFS6 $\mathrm{m}$ is defined as the percentage of patients who are alive and progression-free clinically and radiographically 6 months after the start of an intervention.

\section{Median PFS}

The median PFS is the median survival for a group of patients who remain progression-free after the initiation of an intervention.

\section{Overall survival mOS}

The mOS is defined as the median time from start of treatment to death among a group of trial patients.

\section{2-year survival}

The 2-year survival is defined as the percentage of patients alive 2 years after the beginning of an intervention.

\section{HRQoL}

HRQoL is assessed using validated quality of life instruments such as the European Organisation for the Research and Treatment of Cancer (EORTC) quality of life questionnaires Core-30 and the EORTC brain cancer module (BN-20). ${ }^{22,23}$ The seven domains commonly utilized for the assessment of quality of life changes in trials involving brain tumor patients include global HRQoL and physical role, cognitive, emotional, and social functioning. A $>10$ point change on a 0-100 point scale is considered significant in the determination of changes in HRQoL status. ${ }^{24,25}$ Commonly, quality of life improvements correlate with radiographic response, and deterioration in quality of life correlates with radiographic progression. A drug that is capable of delaying time to tumor progression may therefore favorably impact quality of life in a clinically meaningful way, even if it fails to prolong overall survival. ${ }^{26}$

\section{Current treatment options $n G B M$ and $n A A$}

Patients with nGBM are treated with the three common modalities available for the treatment of all gliomas: surgery, RT, and chemotherapy. The first step in the management of gliomas is to obtain a pathologic diagnosis either through a stereotactic biopsy or open resection. Ideally, the surgeon attempts to perform a gross total resection of the tumor with the exception of deep-seated, inaccessible tumors, and tumors located in eloquent brain regions where resection may result in significant neurologic compromise. Postoperatively, involved field radiation is given to the enhancing region plus a $2-3 \mathrm{~cm}$ margin. RT is typically given in fractions of 2 Gy each for 6 weeks (60 Gy total). ${ }^{27}$ Postoperative RT and chemotherapy are given to treat residual disease that is invariably present following surgery. ${ }^{28}$ 
For patients with nAA, the current standard treatment options include maximal feasible surgical resection followed by involved field, conformal RT similar to the dose schedule prescribed for nGBM (60 Gy in 30 fractions of 2 Gy each). Although there is scant evidence to support a role for adjuvant chemotherapy in the management of AA, many neurooncologists frequently prescribe adjuvant chemotherapy for patients with this disease.

\section{rGBM and rAA}

Patients with GBM invariably exhibit disease recurrence, even among those with a complete response following successful treatment. At the time of disease recurrence, patients are commonly prescribed second-line chemotherapy agents, most often a nitrosourea. ${ }^{29}$ Other chemotherapeutic agents utilized in the salvage setting include nitrosoureas, etoposide, cyclophosphamide, and platinum compounds. ${ }^{30-32}$

A second craniotomy with tumor resection is typically reserved for reasonably well patients with tumors that have caused worsening neurologic deficits or a rise in intracranial pressure. ${ }^{33}$ Repeat irradiation is also a treatment option at the time of relapse but this is seldom prescribed because of the markedly increased risk of radionecrosis and leukoencephalopathy associated with repeat irradiation. ${ }^{34,35}$ Some of the newer RT techniques such as intensity-modulated radiation therapy and radiosurgery may minimize such risks but these remain experimental approaches for recurrent gliomas. ${ }^{36}$

\section{Unmet needs}

Malignant astrocytomas have long been considered amongst the most resistant cancers to therapeutic intervention. Despite advances in surgical and RT techniques over the last few decades, these therapies have had minimal impact on survival statistics. Attempts to improve survival with the addition of adjuvant chemotherapy have generally been disappointing, and numerous large phase III trials examining this matter have failed to show a survival advantage for chemotherapy-treated patients. ${ }^{37-40}$ Despite these generally negative results, a systematic meta analysis of 12 randomized trials demonstrated a small, but statistically significant, survival advantage for patients treated with adjuvant chemotherapy (a $5 \%$ increase in 2-year survival from $15 \%$ to $20 \%)^{41}$

\section{Clinical evidence with TMZ}

TMZ is an orally active alkylating agent that has shown activity in the treatment of malignant gliomas. Unlike many other chemotherapeutic agents, it readily crosses the bloodbrain barrier and has a well tolerated safety profile. Until recently, the nitrosoureas were the first-line agents used for the treatment of malignant gliomas despite their minimal efficacy and significant toxicity. TMZ on the other hand has now become the first-line agent particularly for the treatment of GBM because of its proven benefit and good safety profile.

\section{TMZ pharmacology}

TMZ is a second generation imidazotetrazinone derivative. It undergoes a series of spontaneous reactions under physiologic conditions beginning with its hydrolysis into its active metabolite 5-(3-methyl-1-triazen-1-yl) imidazole4-carboxamide, which further undergoes degradation into a methyldiazonium ion. This ion is the active compound that transfers a methyl group to DNA and thus affects the cytotoxic activity of TMZ. Although the final degradation product, 4-amino-5-imidazole-carboxamide is eliminated through the kidney, no TMZ dose adjustments are usually required for patients with impaired renal function. ${ }^{42}$

TMZ exerts its cytotoxic action via DNA methylation at the $\mathrm{N}-7$ and $\mathrm{O}-6$ positions of guanine, and the O-3 position of adenine. Although methylation of the O-6 position of guanine represents only a small fraction of the total DNA lesions induced by TMZ, it remains the major effector of its cytotoxic action. Methylation of guanine at the O-6 position results in "mismatch" incorporation of thymidine instead of cytosine.

This error is recognized by the mismatch repair (MMR) enzyme system that attempts to excise thymidine. Since guanine methylation persists, a series of futile replication and repair cycles ensue, ultimately resulting in apoptotic cell death. In the presence of the DNA repair enzyme O-6-methlguanine-DNA methyltransferase (MGMT, a DNA repair enzyme that removes methyl adducts at the O-6 position of guanine added by TMZ), ${ }^{43}$ the methylation of guanine can be repaired thereby allowing the cells to escape TMZ-induced cell death. Therefore, for TMZ to exert its cytotoxic action, a cell needs an intact MMR system and deficient or low MGMT enzyme levels. Conversely, a deficient MMR system coupled with high MGMT expression levels may mediate TMZ resistance. ${ }^{44,45}$

\section{TMZ toxicity profile}

Drug-related toxicities are typically graded according to the National Cancer Institute Common Toxicity Criteria (NCI-CTC) version 3.0. ${ }^{46}$ The most common toxic side effects associated with TMZ are nausea, vomiting, fatigue, and hematologic toxicity. According to the NCI-CTC, toxic side effects are graded on a scale of $1-5$. Tables 2 and 3 
Table 2 Nonhematologic toxicities associated with TMZ

\begin{tabular}{|c|c|c|c|c|c|}
\hline Grade I & Grade 2 & Grade 3 & Grade 4 & Grade 5 & \\
\hline Nausea & $\begin{array}{l}\text { Loss of appetite without } \\
\text { alteration in eating habits }\end{array}$ & $\begin{array}{l}\text { Decreased oral intake without weight } \\
\text { loss, dehydration or malnutrition; IV } \\
\text { fluids indicated }<24 \text { hrs }\end{array}$ & $\begin{array}{l}\text { Inadequate oral fluids or } \\
\text { caloric intake: IV fluids, tube } \\
\text { feeds or TPN required }>24 \mathrm{hrs}\end{array}$ & $\begin{array}{l}\text { Life-threatening } \\
\text { consequences }\end{array}$ & Death \\
\hline Vomiting & I episode in $24 \mathrm{hrs}$ & $\begin{array}{l}2-5 \text { episodes in } 24 \mathrm{hrs} \text {, IV fluids } \\
\text { required }<24 \mathrm{hrs}\end{array}$ & $\begin{array}{l}>6 \text { episodes in } 24 \mathrm{hrs} \text {, IV fluids } \\
\text { or TPN required }>24 \mathrm{hrs}\end{array}$ & $\begin{array}{l}\text { Life-threatening } \\
\text { complications }\end{array}$ & Death \\
\hline Fatigue & Mild fatigue over baseline & $\begin{array}{l}\text { Moderate fatigue causing difficulty } \\
\text { with performing ADL }\end{array}$ & $\begin{array}{l}\text { Severe fatigue interfering with } \\
\text { ADL }\end{array}$ & Disabling fatigue & \\
\hline
\end{tabular}

Abbreviations: ADL, activities of daily living; IV, intravenous; TPN, total parenteral nutrition.

summarize the various grades of severity of some of the more common side effects associated with TMZ.

There are single case reports describing instances of severe toxic side effects associated with TMZ treatment. One report describes the development of listeria, brain abscess, pneumocystis pneumonia, and Kaposi's sarcoma in a GBM patient treated with TMZ. ${ }^{47}$ Another report describes the development of organizing pneumonitis associated with temozolomide. ${ }^{48}$

\section{Objective radiographic response nGBM and nAA}

TMZ has been assessed in several clinical trials for the treatment of nGBM as a single agent. Response rates varied depending on the dosing schedule utilized in these trials.

A phase II multicenter study examined the role of neoadjuvant TMZ (prior to RT) for the treatment of nGBM and nAA patients. In this trial, a total of 187 patients received two cycles of TMZ at $200 \mathrm{mg} / \mathrm{m}^{2} /$ day for 5 consecutive days every 28 days, and the objective response rate was determined using the bidimensional criteria on pre and postchemotherapy enhanced scans. A total of 162 patients were evaluable for response, and the ORR was $20 \%(95 \%$ CI 14,26$){ }^{49}$

A nonrandomized open-label phase II study evaluated the role of TMZ in combination with the antiangiogenic agent thalidomide for the treatment of nGBM. A total of 44 patients were treated with surgery followed by RT, and subsequently 19 patients (43\%) received thalidomide alone and $25(57 \%)$ received thalidomide with TMZ. Only two patients $(8 \%)$ in the combined thalidomide/ TMZ group achieved an ORR. ${ }^{50}$

A phase I trial assessed the safety and efficacy of combining TMZ with procarbazine (PCB) for the treatment of glioma patients. This trial enrolled $16 \mathrm{nGBM}$, seven nAA, and five low-grade glioma patients. Although this trial was designed to evaluate the safety of the TMZ/PCB combination, a $36 \%$ ORR was observed. ${ }^{51}$

Neoadjuvant TMZ in combination with carmustine was evaluated for the treatment of analplastic gliomas in a phase II trial. A total of 41 patients received four cycles of neoadjuvant TMZ and carmustine prior to RT. Histology included nAA (81\%), newly diagnosed anaplastic oligodendroglioma $(12 \%)$, and mixed tumors (7\%). The ORR was $29 \%$, with a $2 \% \mathrm{CR}$ and a $27 \% \mathrm{PR}^{52}$ Despite a reasonable response rate, this combination chemotherapy regimen was associated with considerable myelosuppression.

\section{rGBM and rAA}

Two pivotal trials have evaluated the response of patients with rGBM to TMZ treatment ${ }^{26,53}$ and one trial has evaluated rAA. ${ }^{54}$ The ORR to TMZ in rGBM in the two trials was $5 \%$ and $8 \%$, respectively, ${ }^{26,53}$ which was similar to an ORR of $6 \%$ among rGBM patients treated with a variety of chemotherapeutic agents including nitrosoureas..$^{55}$ The ORR with

Table 3 Hematologic toxicities associated with TMZ

\begin{tabular}{llllll}
\hline & Grade I & Grade 2 & Grade 3 & Grade 4 & Grade 5 \\
\hline Leucocyte count $\left(10^{9} / \mathrm{L}\right)$ & $<$ LLN-3 & $<3.0-2.0$ & $<2.0-1.0$ & $<1.0$ & Death \\
Lymphocyte count $\left(10^{9} / \mathrm{L}\right)$ & $<$ LLN-0.8 & $<0.8-0.5$ & $<0.5-0.2$ & $<0.2$ & Death \\
Neutrophils $\left(10^{9} / \mathrm{L}\right)$ & $<$ LLN-1.5 & $<1.5-1.0$ & $<1.0-0.5$ & $<0.5$ & Death \\
Hemoglobin $(\mathrm{g} / \mathrm{dL})$ & $<$ LLN-10 & $<10-8$ & $<8-6.5$ & $<6.5$ & Death \\
Platelets $\left(10^{9} / \mathrm{L}\right)$ & $<$ LLN-75 & $<75-50$ & $<50-25$ & $<25$ & Death \\
\hline
\end{tabular}

Notes: $<$ LLN, less than lower limit of normal. 
TMZ among rAA patients, however, was 35\%, ${ }^{54}$ an outcome that compared favorably with a $14 \%$ ORR for patients treated with other chemotherapeutic agents. ${ }^{55}$ This trial served as the foundation for the approval of TMZ in 1999 in Europe and the US for the treatment of rAA. Although objective response rates in rGBM patients treated with TMZ were less impressive than data derived from rAA patients, a study by Yung and colleagues comparing efficacy of TMZ with PCB noted that the overall response (PR + stable disease) was higher among patients treated with TMZ [51/112 patients (45.6\%) versus 37/113 (32.7\%), $P=0.049] .{ }^{26}$ The ORR (PR + CR) however was no different between the two groups, with six PR with both TMZ and PCB, and no CR with either agent. ${ }^{26}$

\section{Progression-free survival $n G B M$ and $n A A$}

TMZ has been shown to increase the percentage of progressionfree patients with malignant gliomas at the 6-month time point following initiation of treatment. Several trials have evaluated the effect of TMZ on PFS6m either alone or in combination with other drugs for the treatment of nGBM and nAA, ${ }^{28,56}$ including a phase II trial that investigated the role of TMZ in combination with lomustine for the treatment of nGBM. Lomustine was administered at a dose of $110 \mathrm{mg} / \mathrm{m}^{2}$ (day 1) with TMZ $100 \mathrm{mg} / \mathrm{m}^{2} /$ day (days 2-6) plus involved field RT to 31 patients with nGBM. The PFS6m for this group was $61.3 \%{ }^{56}$ The role of neoadjuvant TMZ was investigated in another phase II trial where TMZ was given after a biopsy was performed and prior to RT. The ORR to TMZ was therefore assessed without the confounding effects of surgery or radiotherapy. ${ }^{49}$

Several in-vitro studies suggested that TMZ may act as a radiosensitizer when administered concurrently with $\mathrm{RT} .^{57,58}$ In order to investigate whether TMZ administered during and after RT provides a clinical benefit compared with RT alone, a pilot phase II trial was conducted by Stupp et al. ${ }^{59}$ A total of 64 patients with nGBM from two institutions were randomized to receive TMZ $75 \mathrm{mg} / \mathrm{m}^{2} /$ day for 42 consecutive days during RT (60 Gy in 30 fractions), followed by adjuvant TMZ 150-200 mg/m²/day for 5 consecutive days every 28 days. Patients received up to six cycles of adjuvant TMZ following concurrent treatment. Although this trial did not report PFS6m or median progression-free survival (mPFS), a mOS of 16 months, a 1-year survival of 58\%, and a 2-year survival of $31 \%$ were reported. ${ }^{59}$

A similar phase II trial examined the efficacy of TMZ in combination with RT followed by adjuvant TMZ. ${ }^{60}$ In this trial 130 patients (110 assessable) were randomly assigned to receive either TMZ $75 \mathrm{mg} / \mathrm{m}^{2} /$ day with concurrent $\mathrm{RT}$
(60 Gy in 30 fractions; $n=57$ ) followed by six cycles of adjuvant TMZ $150 \mathrm{mg} / \mathrm{m}^{2} /$ day on days $1-5$ and days $15-19$ every 28 days, or RT alone (60 Gy in 30 fractions; $n=53$ ). The administration of TMZ on days 15-19 of each adjuvant cycle represented a 50\% increase in TMZ dose compared to the conventional regimen. The PFS6m observed in this trial was $67.1 \%$ in the TMZ/RT group compared with $44.9 \%$ in the RT only group. The 1-year survival for patients treated with TMZ and RT was 56.3\% (95\% CI 44.12, 71.60), an outcome that was comparable to that observed in the trial conducted by Stupp et al..$^{59}$

The encouraging phase II results using concurrent and adjuvant $\mathrm{TMZ}$ for the treatment of $\mathrm{nGBM}$ led to a definitive phase III trial conducted by EORTC and the National Cancer Institute of Canada Clinical Trials Group (NCIC CTG).$^{28}$ In this large randomized international trial, 286 patients were assigned to receive RT alone and 287 patients were assigned to receive RT plus TMZ followed by adjuvant TMZ for a maximum of six cycles. RT consisted of 60 Gy administered in 30 fractions, and chemotherapy consisted of TMZ administered concurrently during RT at a dose of $75 \mathrm{mg} / \mathrm{m}^{2} /$ day. The adjuvant TMZ regimen was $150 \mathrm{mg} / \mathrm{m}^{2} /$ day for 5 days on cycle 1 and if tolerated, increased to $200 \mathrm{mg} / \mathrm{m}^{2} /$ day for 5 days every 28 days on cycles 2 and thereafter. Patients receiving concurrent and adjuvant TMZ had a significantly better PFS6m (53.9\%, CI 48.1, 59.6), compared with those receiving RT alone $(36.4 \%$, CI $30.8,41.9){ }^{28}$

\section{rGBM and rAA}

Three pivotal phase II trials have established a role for TMZ in the treatment of patients with rGBM and rAA. ${ }^{26,53,54}$ These trials were the first to adopt PFS6m as a primary endpoint. Earlier trials utilizing the ORR as an endpoint may have underestimated clinical efficacy because clinical and quality of life benefits may be derived without radiographic evidence of tumor regression. ${ }^{17,18}$ The first two trials evaluated the efficacy of TMZ in rGBM and showed a PFS6m of $18 \%{ }^{53}$ and $21 \%$ (versus $8 \%$ for $\mathrm{PCB})^{26}$ respectively, and a corresponding ORR of only $8 \%{ }^{53}$ and $5 \% .{ }^{26}$ Since a PFS $6 \mathrm{~m}$ of $>10 \%$ was deemed indicative of adequate drug activity, ${ }^{61,62}$ these trials demonstrated a positive role for TMZ in rGBM. TMZ was subsequently approved in Europe for the treatment of rGBM.

A third trial evaluating TMZ in rAA enrolled 162 patients and demonstrated an impressive PFS6m of $46 \% .{ }^{54}$ The ORR among this group of rAA patients was 35\%, results that compared favorably with an MD Anderson Cancer Center database of rAA patients who received various chemotherapeutic agents and had a PFS6m of only $31 \% .{ }^{55}$ 
This led to the accelerated approval of TMZ in 1999 by the FDA and the European Medicines Agency (EMEA) for the treatment of rAA.

TMZ has also been investigated in combination with other chemotherapeutic and targeted agents in an attempt to improve its efficacy against malignant gliomas. A recent North American Brain Tumor Consortium phase II trial combined TMZ with the antiangiogenic agent thalidomide for rGBM patients. ${ }^{63}$ This trial enrolled 44 rGBM patients (43 patients were evaluable) for clinical efficacy and safety of TMZ. Eligible patients had rGBM after standard surgical resection and RT, and $82 \%$ of patients were chemotherapy-naïve at the time of relapse. TMZ was given in the standard 5-day cycle (150-200 $\mathrm{mg} / \mathrm{m}^{2} /$ day on days $1-5$ every 28 days) and thalidomide $400 \mathrm{mg}$ was given orally on days $1-28$, and escalated to $1200 \mathrm{mg}$ if tolerated. This trial demonstrated a PFS6m of $24 \%$, suggesting that the combination was not superior to single agent TMZ when used for the treatment of rGBM. ${ }^{63}$

MGMT has been implicated as one of the mechanisms of tumor resistance to alkalytator and methylator chemotherapies. ${ }^{44,45}$ Depletion of MGMT has been demonstrated in vitro by cisplatin and continuous TMZ administration. ${ }^{64}$ TMZ in combination with cisplatin was therefore evaluated for the treatment of rGBM patients. ${ }^{65}$ In this phase II study, 50 patients with rGBM (49 patients were assessable) were given TMZ $130 \mathrm{mg} / \mathrm{m}^{2}$ bolus on day 1 , followed by $70 \mathrm{mg} / \mathrm{m}^{2}$ every 12 hours on days $2-5$, repeated every 4 weeks. Cisplatin was given in a dose of $75 \mathrm{mg} / \mathrm{m}^{2}$ on day 1 only of each 4-week cycle. The dose of TMZ was escalated to a cumulative maximum of $1000 \mathrm{mg} / \mathrm{m}^{2}$ over 5 days in the absence of hematologic toxicity. This regimen resulted in a PFS6m of $34 \%(95 \%$ CI 23,50$)$ and a median PFS of 18.4 weeks, and was associated with an acceptable toxicity profile. ${ }^{65}$

Another phase II trial evaluated a TMZ and cisplatin combination regimen in rGBM and rAA patients. ${ }^{66}$ Thirty-three patients (20 rGBM and 13 rAA patients) were treated with cisplatin $40 \mathrm{mg} / \mathrm{m}^{2}$ intravenously on days 1 and 2 plus TMZ $200 \mathrm{mg} / \mathrm{m}^{2} /$ day on days 2-6 (24 hours after first cisplatin dose), and cycles were repeated every 4 weeks. This regimen resulted in a 52\% PFS6m and a mPFS of 33 weeks (29.3 weeks for GBM and 39.5 weeks for AA). However, the higher PFS6m and mPFS demonstrated in this trial is likely due to the proportion of rAA patients included. ${ }^{66}$

Liposomal doxorubicin has demonstrable activity in a variety of malignancies, ${ }^{67}$ and its combination with TMZ was recently explored for the treatment of rGBM. ${ }^{68}$ In this phase II trial, 22 patients with rGBM were given liposomal doxorubicin $40 \mathrm{mg} / \mathrm{m}^{2}$ on day 1 with TMZ $200 \mathrm{mg} / \mathrm{m}^{2} /$ day on days 1-5 administered in 4-week cycles. This regimen produced a PFS $6 \mathrm{~m}$ of $32 \%$ and an mPFS of 3.2 months. ${ }^{68}$

The matrix metalloproteinase inhibitor marimastat has also been investigated in combination with TMZ for the treatment of rGBM. Evidence derived from in-vitro data indicated that marimastat can inhibit glioma cell invasion, and for this reason its combination with TMZ is theoretically appealing. ${ }^{69}$ A total of 44 patients with $\mathrm{rGBM}$ received TMZ (200 mg/m²/day, days $1-5$ ) and marimastat ( $50 \mathrm{mg} /$ day, days $8-28)$ in a 28-day cycle for two cycles. The PFS6m in this group of patients was $39 \%$ and mPFS was 17 weeks. This study was complicated by a $47 \%$ incidence of joint and tendon pain that resulted in the removal of $11 \%$ of patients from the study. The authors concluded that while the activity of this regimen appeared promising, further refinements were needed to minimize the toxic side effects. ${ }^{70}$

TMZ maintains activity in rGBM even at second recurrence. A small Italian study demonstrated a PFS6m of 24\% when TMZ (standard 5-day regimen) was given to a group of 42 patients with rGBM at the time of second relapse. ${ }^{71}$ Although these patients were heavily pretreated with various chemotherapeutic agents, TMZ remained a viable therapeutic option for this group of patients.

Continuous daily dosing or "dose-dense" TMZ represents an attractive treatment approach based on in-vitro data demonstrating rapid MGMT enzyme depletion with continuous exposure to TMZ. ${ }^{72}$ A small Memorial Sloan-Kettering Cancer Center phase II trial evaluated continuous TMZ in recurrent malignant gliomas. This trial enrolled 35 patients (28 with rGBM, three with rAA, two with recurring anaplastic oligodendroglioma, and two with recurring anaplastic oligoastrocytomas) to receive TMZ $75 \mathrm{mg} / \mathrm{m}^{2} /$ day for 42 days, in 70-day cycles. The PFS6m for the entire group was $27 \%$, while PFS6m for the subgroup of GBM patients was $19 \% .^{73}$ Despite the acceptable PFS6m, the data failed to show superiority of this approach over the standard 5-day TMZ regimen.

\section{Overall survival $\mathrm{nGBM}$ and $\mathrm{nAA}$}

The definitive EORTC/NCIC phase III trial has demonstrated conclusively that TMZ can prolong mOS in $\mathrm{nGBM}$ patients by 2.5 months. ${ }^{28}$ These results confirm earlier phase II trials that demonstrated a survival advantage for TMZ in newly diagnosed malignant astrocytomas. ${ }^{56,60} \mathrm{TMZ}$ given immediately in the postoperative setting and prior to neoadjuvant RT for $\mathrm{nGBM}$ and $\mathrm{nAA}$ patients resulted in a mOS of 10 months (16 months for responding patients 
versus only 3 months for nonresponders). ${ }^{49}$ Assessing TMZ efficacy in the neoadjuvant setting provides an advantage in that the benefit derived is not confounded by an RT effect or the effect of other chemotherapeutic agents.

The promising results obtained with neoadjuvant $\mathrm{TMZ}$ treatment led to further refinements of TMZ dosing schedules for the treatment of nGBM. A pilot phase II trial conducted by Stupp and colleagues ${ }^{59}$ assessed the novel approach of concurrent TMZ/RT followed by adjuvant TMZ given to 69 nGBM patients. ${ }^{59}$ The mOS reported in this study was 16 months. Another phase II trial compared $\mathrm{TMZ}+\mathrm{RT}$ followed by adjuvant $\mathrm{TMZ}\left(150 \mathrm{mg} / \mathrm{m}^{2} /\right.$ day on days 1-5 and 15-19) with RT alone. The mOS reported for the TMZ/RT group was 13.4 versus 7.7 months for the RT group $(P=0.001) .{ }^{59}$ The phase II data reported by Stupp et $\mathrm{al}^{59}$ led to a definitive phase III trial conducted by the EORTC/NCIC in order to confirm the benefit of this novel regimen for the treatment of nGBM patients. ${ }^{28}$ As noted earlier, this trial enrolled 573 patients (286 patients in the RT arm and 287 patients in the TMZ/RT arm) most of whom were pathologically-proven GBM patients $(93 \%$ in the RT arm and $92 \%$ in the TMZ/RT arm). At a median follow up of 28 months, $84 \%$ of the enrolled patients had died. For the patients who received concurrent and adjuvant TMZ, their unadjusted hazard ratio for death was 0.63 (95\% CI 0.52 , $0.75 ; P<0.007$ by the log-rank test), which translates into a $37 \%$ reduction in the risk of death for nGBM patients receiving concurrent and adjuvant $\mathrm{TMZ}$, compared with those receiving RT alone. The mOS reported for the TMZ/RT group was 14.6 (95\% CI 13.2, 16.8) versus 12.1 months (95\% CI 11.2, 13.0) for RT alone, which translates into a 2.5-month increase in mOS for the TMZ/RT group compared with the RT only group. This was the first phase III trial to demonstrate a statistically significant and clinically meaningful survival advantage with the addition of chemotherapy to standard RT for nGBM patients.

Several other studies have used TMZ with other agents in an attempt to further improve the survival statistics. A phase II study enrolling 45 patients (38 with GBM and seven with AA) combined TMZ $\left(150 \mathrm{mg} / \mathrm{m}^{2} /\right.$ day on days $1-5)$ with irinotecan $\left(150 \mathrm{mg} / \mathrm{m}^{2} /\right.$ day on days 6 and 17$)$ given every 4 weeks for a maximum of six cycles. ${ }^{74}$ Patients with GBM had a mOS of 12.8 months but this regimen was associated with a high rate of toxicity and failed to improve the survival statistics over TMZ alone for the treatment of nGBM and nAA patients.

Another phase II trial treated 31 patients with a combination of involved field RT (60 Gy in 30 fractions), lomustine $\left(100 \mathrm{mg} / \mathrm{m}^{2}\right.$ on day 1$)$, and TMZ $\left(100 \mathrm{mg} / \mathrm{m}^{2} /\right.$ day on days 2-6) every 4 weeks for up to six cycles. The reported mOS was 22.6 months and the regimen was associated with acceptable toxicity, indicating that further investigation of this approach may be warranted. ${ }^{56}$

\section{rGBM and rAA}

TMZ has established activity in the treatment of rGBM and rAA patients. Three pivotal phase II studies demonstrated radiographic response to $\mathrm{TMZ}$ in $\mathrm{rGBM}$ and $\mathrm{rAA}$ patients but failed to prolong mOS compared with historic databases of patients treated with other chemotherapeutic agents. ${ }^{26,53-55}$

The first study enrolled 138 rGBM patients and reported an mOS of 5.4 months. ${ }^{53}$ Patients treated with a variety of chemotherapeutic agents in a study conducted by Wong and colleagues $^{55}$ had a similar mOS of 5.8 months. Another trial enrolled 225 rGBM patients randomized to $\mathrm{PCB}$ or TMZ. Patients receiving TMZ treatment had a mOS of 7.3 months compared with 5.8 months in those treated with PCB. This 1.5-month increase in mOS for TMZ-treated patients failed to reach statistical significance. ${ }^{26} \mathrm{~A}$ further trial examining the role of TMZ in rAA enrolled 162 patients and reported a mOS of 13.6 months. ${ }^{54}$ Data derived from the trial conducted by Wong and colleagues of patients receiving a variety of chemotherapeutic agents reported a mOS of 11 months among rAA patients. ${ }^{55}$ Despite the failure of TMZ to improve survival outcome, these trials nevertheless demonstrated improvements in the novel endpoint PFS6m as well as HRQoL, leading to the approval of TMZ for the treatment of rGBM and rAA patients.

Combining TMZ with other agents has also been investigated for the treatment of rGBM and rAA patients. For example, a small trial in 22 patients with rGBM reported a mOS of 8.2 months for the combination of doxorubicin with TMZ. ${ }^{68}$ Another study combining marimastat with TMZ reported a mOS of 45 weeks. ${ }^{70}$

To date, combining TMZ with other agents does not appear to confer an additional survival advantage over TMZ alone for the treatment of recurrent malignant astrocytomas. Table 4 summarizes the effect of TMZ on various trial endpoints when used for the treatment of malignant astrocytomas.

\section{HRQoL}

The addition of TMZ to the standard treatment approaches of surgery and RT has been shown to impact positively on HRQoL among recurrent glioma patients. Two studies by 
Osoba and colleagues ${ }^{75,76}$ examined the effect of TMZ on HRQoL in patients with rAA and rGBM.

The first study calculated changes from baseline scores of seven HRQoL domains (role and social functioning, global quality of life, visual disorder, motor disorder, communication deficit, and drowsiness) in rAA patients treated with TMZ. ${ }^{76}$ These HRQoL domains were assessed every 4 weeks at each TMZ treatment cycle, and changes were compared to pretreatment (baseline) scores. This study demonstrated a significant improvement (defined as an increase $\geq 10$ points from baseline scores) in all HRQoL domains following TMZ treatment. These improvements correlated with freedom from disease progression, and dropped sharply at the time of disease progression. ${ }^{76}$ Since TMZ is known to delay the time to tumor progression among recurrent glioma patients, this may in part explain the improvements in HRQoL scores seen with TMZ treatment.

A large phase II trial comparing the efficacy of TMZ with PCB in rGBM also included assessments of HRQoL scores. In this trial, 225 patients were randomly assigned to receive either TMZ $(n=112)$ or PCB $(n=113)$ at the time of first relapse. ${ }^{26}$ Patients receiving TMZ scored consistently higher on seven HRQoL domains, and this improvement was maintained until disease progression. Since TMZ was given on every 5 of 28 days while PCB was given daily for 28 days, the authors commented that the improvement in HRQoL may have been related to toxicity associated with daily PCB administration rather than to TMZ benefit. However, the study also demonstrated that TMZ delayed time to tumor progression compared with $\mathrm{PCB}$, a parameter that closely correlates with maintenance or improvement in HRQoL. ${ }^{26}$ Whether or not TMZ directly impacts HRQoL parameters, its favorable toxicity profile compared with other chemotherapeutic agents likely plays a role in maintaining quality of life in a population that has to endure many potential adverse drug events.

Patients enrolled in a recent EORTC/NCIC phase III trial examining the role of concurrent TMZ/RT followed by adjuvant TMZ among nGBM patients were also assessed for changes in the predefined HRQoL measures at baseline and every 3 months following treatment until disease progression. Changes in HRQoL were calculated among patients receiving concurrent TMZ/RT followed by adjuvant TMZ and those receiving RT alone. HRQoL was a secondary endpoint for this study and was calculated based on the EORTC quality of life questionnaire Core- 30 and the EORTC BN-20. The parameters used to measure HRQoL

Table 4 Evidence for TXZ efficacy in malignant astrocytomas

\begin{tabular}{|c|c|c|c|}
\hline & nGBM/nAA & rGBM/rAA & Reference \\
\hline ORR & $\begin{array}{l}\text { Neoadjuvant TMZ: } 20 \% \\
\text { TMZ + TLD: } 8 \% \\
\text { TMZ (GBM,AA and LGG): } \\
36 \%\end{array}$ & $\begin{array}{l}\text { Single agent TMZ for rGBM: } 5 \% \\
\text { Single agent TMZ for rGBM: } 8 \% \\
\text { Single agent TMZ for rAA: } 35 \% \\
\text { rGBM: } 6 \% \\
\text { rAA: } 14 \%\end{array}$ & $\begin{array}{l}\text { Brada et al } ;{ }^{49} \text { Yung et a }\left.\right|^{26} \\
\text { Baumann et al }{ }^{53}{\text { Brada et } \mathrm{a}^{50}} \\
\text { Newlands et al } ;{ }^{51} \text { Yung et a }{ }^{54} \\
\text { Wong et a }\left.\right|^{55}\end{array}$ \\
\hline PFS6m & $\begin{array}{l}\text { TMZ/RT + adjuvant TMZ: } \\
67.1 \% \\
\text { RT alone: } 44.9 \% \text { TMZ/RT + } \\
\text { adjuvant TMZ: } 53.9 \% \\
\text { RT alone: } 36.4 \%\end{array}$ & $\begin{array}{l}\text { rGBM: } 18 \% \\
\text { rGBM: } 21 \% \text { vs } 8 \% \text { for PCB } \\
\text { rAA: } 46 \% \\
\text { rGBM: } 15 \% \\
\text { rAA: } 31 \%\end{array}$ & $\begin{array}{l}\text { Athanassiou et } \mathrm{a}^{60} \\
\text { Brada et } \mathrm{a}^{54} \\
\text { Yung et } \mathrm{a}^{26,54} \\
\text { Stupp et }\left.\mathrm{a}\right|^{28} \\
\text { Wong et } \mathrm{al}^{55}\end{array}$ \\
\hline mOS & $\begin{array}{l}\text { nGBM/nAA (neoadjuvant } \\
\text { TMZ): } 10 \text { mo } \\
\text { nGBM (TMZ/RT + TMZ): } \\
16 \text { mo nGBM } \\
\text { (TMZ/RT + TMZ): I } 3.4 \text { mo } \\
\text { RT alone: } 7.7 \text { mo } \\
\text { nGBM (TMZ/RT + TMZ): } \\
\text { I4.6 mo } \\
\text { RT alone: I2.I mo }\end{array}$ & $\begin{array}{l}\text { rGBM: } 5.4 \text { mo } \\
\text { rGBM: } 7.3 \text { mo vs } 5.8 \text { mo for } \\
\text { PCB } \\
\text { rAA: } 13.6 \text { mo }\end{array}$ & $\begin{array}{l}\text { Brada et al }{ }^{49,50} \text { Stupp et al }{ }^{58} \\
\text { 2002; Yung et al:;6 Athanasiou, } \\
\text { et al:;4 Yung et al }{ }^{108} \\
\text { Stupp et a }\left.\right|^{28}\end{array}$ \\
\hline
\end{tabular}

Notes: a Historic database of patients treated with multiple chemotherapeutic agents in a trial conducted by Wong and colleagues ${ }^{55}$ is used for comparison. bData converted from weeks to months for ease of comparison using the formula (weeks $\times 7$ ) $/ 30=$ months.

Abbreviations: Car, carmustine; Cis, cisplatin; GBM, glioblastoma; LGG, low grade glioma; mo, months; mOS, median overall survival; $\mathrm{nAA}$, newly diagnosed anaplastic astrocytoma; nGBM, newly diagnosed GBM; ORR, objective radiographic response; PCB, procarbazine; PFS6m, progression-free survival at 6 mo; rGBM, recurrent GBM; rAA, recurrent anaplastic astrocytoma; RT, radiotherapy;TLD, thalidomide; TMZ, temozolomide. 
were fatigue, overall health, social function, emotional function, future uncertainty, insomnia, and communication deficit. Improvement in HRQoL was defined as an increase of $\geq 10$ points compared with baseline. Overall, the study concluded that the addition of TMZ to RT did not impair the HRQoL measures compared with RT alone. ${ }^{77}$

\section{Safety and tolerability}

TMZ therapy has been consistently shown to be relatively safe and reasonably well tolerated in multiple trials for the treatment of malignant gliomas. The most commonly tested dosing schedule is a standard 5-day regimen of 150-200 $\mathrm{mg} / \mathrm{m}^{2} /$ day, which was developed in the early 1990 s by Newlands and coworkers. ${ }^{78}$ In an early phase I trial, $\mathrm{TMZ}$ given as asingle bolus of $50 \mathrm{mg} / \mathrm{m}^{2}$, and subsequently escalated to $1200 \mathrm{mg} / \mathrm{m}^{2}$, resulted in no antitumor activity but was associated with myelosuppression as a major doselimiting toxicity. ${ }^{51}$ The 5-day regimen was subsequently developed and given as a starting dose of $150 \mathrm{mg} / \mathrm{m}^{2} /$ day for 5 days, in 28-day cycles and escalated to $200 \mathrm{mg} / \mathrm{m}^{2} /$ day for 5 days on subsequent cycles if tolerated. This regimen was associated with clinical activity as opposed to the single TMZ dose and furthermore, 17\% (two CR and two PR) of 23 patients demonstrated an ORR. ${ }^{51}$ The side effects associated with this regimen included nausea and vomiting that were controlled with $5 \mathrm{HT}_{3}$-antagonists. There was also little cumulative toxicity with this regimen and patients tolerated repeated cycles for up to 3 years. ${ }^{51}$

The first trial to examine the safety and tolerability of TMZ given concurrently and adjuvantly with RT was conducted by Stupp and colleagues in 2002. During the concomitant phase of treatment, grade 111 or IV neutropenia or thrombocytopenia was observed in four patients $(6 \%)$ each. Grade 111 or IV lymphocytopenia was a very common side effect observed with this regimen, with $79 \%$ of patients suffering from this toxicity during the concurrent TMZ/RT phase of treatment. Three patients developed infections: two had Pneumocystis carinii pneumonia (PCP), and one developed osteomyelitis. Both patients with PCP had grade 111 or $1 \mathrm{~V}$ neutropenia and lymphocytopenia at the time of the infection. ${ }^{58}$ Following the development of PCP in these two patients, pentamidine prophylaxis was introduced in the trial to be given to patients receiving concurrent treatment and no further PCP episodes were observed. During the adjuvant phase of treatment grade 111 or IV neutropenia occurred in $2 \%$ of patients, while grade 111 or $1 \mathrm{~V}$ thrombocytopenia occurred in $6 \%$ of patients. Only $2 \%$ of patients developed grade 111 or $\mathrm{IV}$ anemia during adjuvant TMZ administration.
Nonhematologic toxicities during both phases of treatment were well tolerated and included nausea and vomiting (managed by standard antiemetics), moderate to severe fatigue, and rash resulting in discontinuation of treatment. One patient developed intracranial hypertension, refractory seizures, and loss of vision, all of which were considered delayed radiation-induced adverse events rather than side effects related to TMZ.

The EORTC/NCIC phase III trial enrolled the largest number of patients to date treated with TMZ. ${ }^{28}$ It therefore provided a large sample size for assessing safety and tolerability of TMZ in combination with RT and as a single agent in the adjuvant setting. There were no grade 111 or IV hematologic toxic effects noted among those treated with RT alone. During the concurrent RT/TMZ treatment, 12 patients $(4 \%)$ experienced grade 111 or $1 \mathrm{~V}$ neutropenia while grade 111 or $1 \mathrm{~V}$ thrombocytopenia occurred in nine patients $(3 \%)$. Grade 111 or $1 \mathrm{~V}$ hematologic toxicities were reported in 19 patients $(7 \%)$ (Table 5). ${ }^{28}$

Overall, TMZ given concurrently with RT or as a single agent is well tolerated, with noncumulative myelotoxicity as the main dose-limiting side effect. Nonhematologic toxicities are usually well tolerated and include mild-to-moderate fatigue, constipation, nausea, and vomiting.

\section{Prognostic factors}

Prognostic determinants of survival for patients with malignant astrocytomas include tumor grade and histology (ie, GBM or AA), age, Karnofsky performance status (KPS), and MGMT promoter methylation status..$^{79,80}$ The most important prognosticfactor and the one that most closely predicts mOS is tumor grade. Patients with GBM (WHO grade IV) fare worse than those with the lower grade AA (WHO grade III). ${ }^{1}$ Although GBM occurs on average among patients 10 years older than those with AA, age is likely to be an independent negative prognostic factor ${ }^{1}$ Among elderly patients ( $>75$ years) with GBM, the 2-year survival is only $1.4 \%$ compared with $29.8 \%$ for patients aged between 20 and 44 years old. ${ }^{1}$ Patients with a lower performance status fare worse than those who are fully functional, and have a better KPS.

Several trials have evaluated the role of the extent of surgical resection in prolonging survival among malignant astrocytoma patients. ${ }^{81,82}$ Although some trials have shown that patients who have undergone gross total resection have better outcomes compared with those undergoing subtotal resection or biopsy only, these trials are fraught with confounding variables. ${ }^{83,84}$ For example, patients who underwent biopsy only were typically of older age and with worse KPS scores. 
Table 5 Grade 3 or 4 hematologic toxic effects [\% (n)] in patients treated with temozolomide (adapted from Stupp et al ${ }^{28}$ )

\begin{tabular}{llll}
\hline & Concomitant TMZ/RT $(\mathbf{n}=\mathbf{2 8 4})$ & Adjuvant TMZ $(\mathbf{n}=\mathbf{2 2 3})$ & Entire study period $^{\text {a }}(\mathbf{n}=\mathbf{2 8 4})$ \\
\hline Leukopenia & $7(2)$ & II (5) & $20(7)$ \\
Neutropenia & $12(4)$ & $9(4)$ & $21(7)$ \\
Thrombocytopenia & $9(3)$ & $24(11)$ & $33(12)$ \\
Anemia & $1(<1)$ & $2(1)$ & $4(1)$ \\
Any & $19(7)$ & $32(14)$ & $46(16)$ \\
\hline
\end{tabular}

Notes: ${ }^{\text {T}}$ The entire study period is defined as the period from study entry to 7 days after disease progression.

Abbreviations: $\mathrm{RT}$, radiotherapy; TMZ, temozolomide.

In such instances, multivariate analysis revealed that the extent of surgery did not correlate with improved survival. ${ }^{85}$

Curran and colleagues ${ }^{86}$ have developed a recursive partitioning analysis (RPA) model of treatment and pretreatment-related prognostic variables for patients with malignant gliomas ${ }^{86,87}$ (Table 6).

The RPA model was applied to the 64 patients enrolled in the phase II trial comparing RT with RT/TMZ for nGBM patients. ${ }^{87}$ Median survival among these patients was 24 months for RPA class III, 14 months for RPA class IV, and 9 months for RPA class V, which compared favorably with a Radiation Therapy Oncology Group database. ${ }^{87}$ This was an incentive for conducting a larger phase III randomized trial that resulted in a new standard of care for the treatment of nGBM. ${ }^{28}$ The RPA methodology in this study was also used to evaluate the effect of adding TMZ to RT on median survival time. Among patients in the RPA class III receiving $\mathrm{RT}+\mathrm{TMZ}$, there was a gain of 7 months in median survival time and of $24 \%$ in the 2 -year survival time $(P=0.006)$. For this subset of patients, adding TMZ to RT resulted in a $43 \%$ probability of survival at 2 years. ${ }^{28}$ The addition of TMZ to RT still maintained its benefit among RPA class IV patients, more than doubling the 2-year survival rates compared with RT alone $(P=0.0001)$. The gain among the RPA class $\mathrm{V}$ patients was statistically insignificant $(P=0.054)$, indicating that such patients with poor prognostic variables are least likely to benefit from addition of TMZ to RT (Table 7). ${ }^{28}$ However, this should not be taken as a reason to withhold TMZ treatment from RPA class V patients, since it is not known whether they derive benefits in other variables such as HRQoL.

\section{Ongoing clinical development}

Several trials are ongoing to evaluate the efficacy of $1 \mathrm{TMZ}$ with various chemotherapeutic as well as targeted agents. For example, a phase I trial evaluating the safety and tolerability of adding everolimus (RAD001), an inhibitor of mammalian

Table 6 Original and adapted RTOG/EORTC RPA class III-V (adapted from Mirimanoff et $\mathrm{al}^{87}$ )

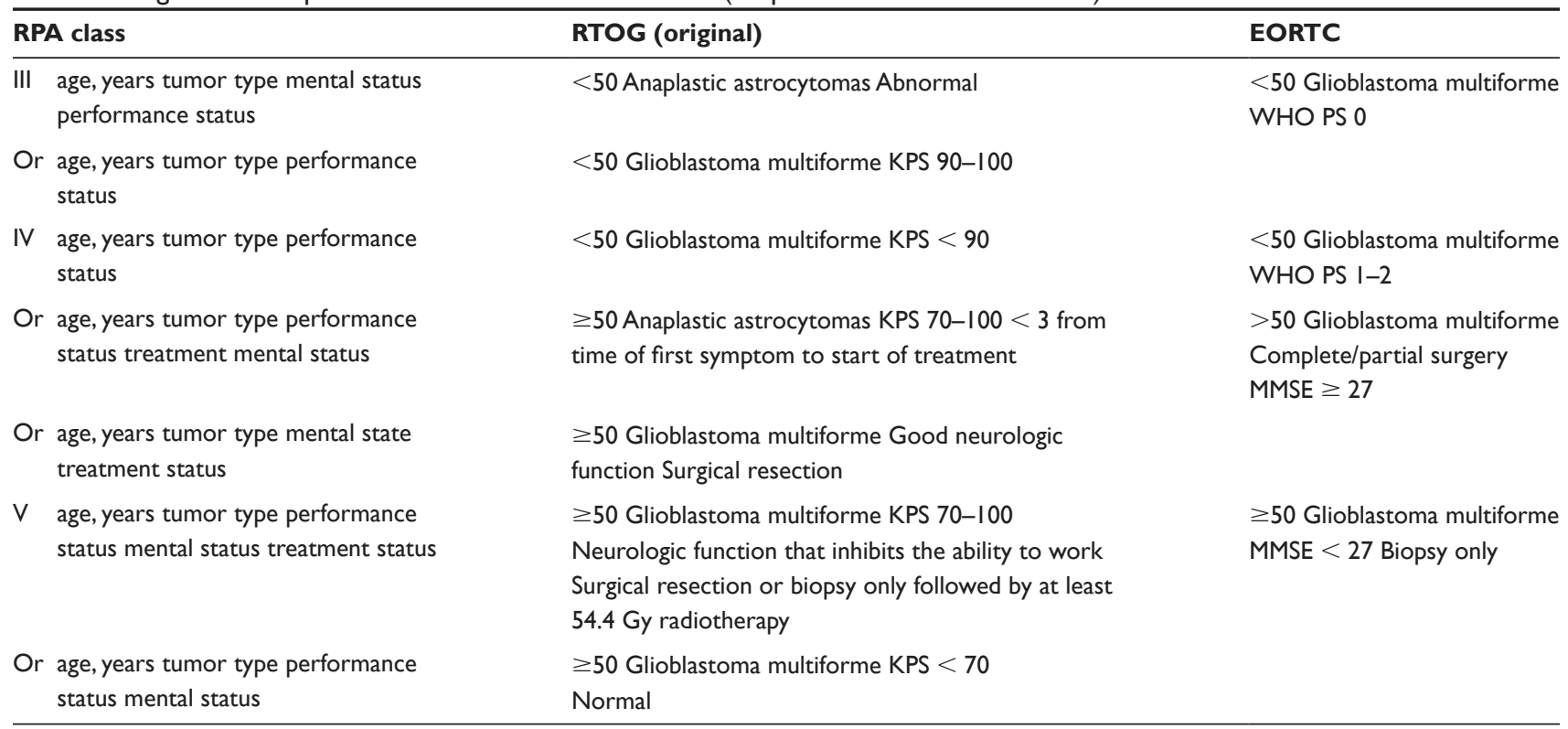

Abbreviations: EORTC, European Organization for Research and Treatment of Cancer; KPS, Karnofsky performance status; MMSE, Mini Mental State Evaluation; RPA, recursive partitioning analysis; RTOG, Radiation Therapy Oncology Group; WHO PS, World Health Organization performance status. 
target of rapamycin (mTOR), to TMZ in nGBM patients is currently being conducted by the NCIC CTG. ${ }^{88}$

Continuous daily "dose-dense" TMZ is also currently under investigation for the treatment of recurrent malignant gliomas. Patients with recurrent malignant gliomas who have shown evidence of progression on the 5-day TMZ regimen are candidates for this trial. Patients receive TMZ at $75 \mathrm{mg} / \mathrm{m}^{2} /$ day in 28 -day cycles, and the cycles are repeated for up to a year if tolerated. ${ }^{89}$

In order to overcome MGMT-induced TMZ resistance, some trials have assessed the efficacy of combination regimens with TMZ and O-6-benzylguanine (an MGMT inhibitor). ${ }^{90,91}$ To date, these trials have failed to demonstrate improvements in outcome compared with $\mathrm{TMZ}$ alone but further investigations are ongoing.

\section{Economic evidence}

Because TMZ is associated with high acquisition costs, it is important to assess whether these additional costs are associated with measurable benefits. A report by Wasserfallen and colleagues ${ }^{92}$ showed that the addition of TMZ to RT for the treatment of nGBM patients was eight times more expensive than RT alone. ${ }^{92}$ Several reports therefore assessed the costs of TMZ per life-year and QALY gained, and whether such costs were below the acceptable threshold of \$US50 000 per life-year gained as proposed by Prosser and colleagues. ${ }^{92}$ The decision whether or not to reimburse TMZ treatment by payors may be influenced by such economic analyses of cost-1 ratios, especially in systems with limited healthcare resources.

Data from a phase II trial evaluating the safety and efficacy of concurrent and adjuvant TMZ in nGBM. ${ }^{59}$ were collected, and associated costs retrospectively computed. ${ }^{92}$ The phase II trial enrolled 64 patients from two institutions, but only data related to 46 patients at one institution (University Center of Vaud, Lausanne, Switzerland) were analysed. Costs were computed for four separate periods: during concurrent TMZ and RT; during adjuvant TMZ; during the follow-up period and up to disease recurrence; and finally from disease recurrence until death.

Table 7 Overall survival rates by RPA class (adapted from Stupp et $\mathrm{al}^{28}$ )

\begin{tabular}{llllll}
\hline RPA class & \multicolumn{2}{l}{ Median survival } & & \multicolumn{2}{c}{ 2-year survival } \\
\cline { 2 - 3 } \cline { 6 - 7 } & Months & $\mathbf{9 5 \%} \mathbf{~ C l}$ & & $\%$ & $\mathbf{9 5 \%} \mathbf{~ C l}$ \\
\hline III & 17 & $15-2 \mid$ & 32 & $21-2$ \\
IV & 15 & $13-16$ & & 19 & $15-24$ \\
V & 10 & $9-12$ & & 11 & $7-16$ \\
\hline
\end{tabular}

Abbreviation: RPA, recursive partitioning analysis.
This study showed that TMZ treatment had an average cost of $€ 20952$ (eight times more expensive than RT alone). TMZ drug acquisition costs represented $54 \%$ of the total costs during the concurrent phase and $75 \%$ of the total costs during the adjuvant phase. ${ }^{93}$ Costs associated with the followup period (after completion of adjuvant TMZ) were largely attributable to hospitalizations as well as brain imaging studies, while those associated with disease recurrence were mainly due to palliative care hospitalizations and further chemotherapy treatments. This study also reported that the overall cost of care associated with TMZ use for nGBM patients treated in this trial ranged from $€ 10893$ to $€ 125275$, with a median of $€ 34362$, and that TMZ acquisition costs represented $55 \%$ of this sum. The median cost per month of survival was $€ 2307$ and the cost of TMZ per year of survival was $€ 27$ 684. Interestingly, tumor MGMT promoter status and extent of surgery significantly affected the costs associated with TMZ treatment. Patients with tumors that had methylated MGMT promoter had a statistically significantly longer follow-up period before disease recurrence compared with patients with tumors that had unmethylated MGMT promoter $(19.6 \pm 17.7$ versus $2.3 \pm 1.7$ months; $P=0.049)$. The costs incurred during this longer follow-up period were higher ( $€ 4793 \pm 3848$ versus $€ 636 \pm 633 ; P=0.049$ ), but the average monthly costs were similar. Patients who underwent surgical debulking also fared better than those who underwent biopsy only, and had longer adjuvant periods of TMZ treatment (144 \pm 50 versus $80 \pm 24$ days, $P=0.032$ ) and longer survival $(10.9 \pm 10.7$ versus $4.1 \pm 4.7$ months, $P=0.014)$. Interestingly, the total costs per month of survival for patients undergoing surgical debulking were lower than in those undergoing biopsy only because they required fewer hospitalizations despite the greater costs associated with TMZ use. ${ }^{92}$ This study was limited, however, by the small sample size and the retrospective nature of the analysis. Moreover, cost-effectiveness or cost-utility measures for $\mathrm{TMZ}$ in nGBM patients were not determined. Importantly, this study demonstrated that since concurrent and adjuvant TMZ was associated with a 4-month increase in median survival, the additional costs associated with this regimen would exceed the acceptable threshold of \$US50 000 per life-year gained. The authors argued that this should not be a reason to withhold TMZ from a patient population with little or no other effective alternatives.

Another report by Wasserfallen and colleagues ${ }^{94}$ calculated the cost effectiveness and cost utility of TMZ use in patients with rGBM or rAA. The median cost effectiveness of TMZ in this patient population ranged between $€ 28817$ and 
$€ 38450$. When these cost s were weighted by the individual mean health status (area under the Karnofsky curve), the cost utility ranged from $€ 41167$ to $€ 53369$ per QALY gained. This figure fell below the accepted \$US50 000 threshold. ${ }^{94}$

A report commissioned by the Health Technology Assessment Programme on behalf of the National Institute for Health and Clinical Excellence (NICE) in England and Wales, reviewed the cost effectiveness of TMZ based on a literature review up to the year $2000^{95}$ (prior to the completion of the EORTC/NCIC phase III trial). The report concluded that since TMZ resulted in gains in PFS but not in overall survival for patients with rGBM, the cost per QALY gained was approximately $€ 40000$. Furthermore, this report concluded that until appropriate randomized controlled trials comparing $\mathrm{TMZ}$ with other alternative therapies were conducted, no firm conclusions about the cost effectiveness of TMZ should be drawn. ${ }^{97}$

However, the more recent EORTC/NCIC phase III trial clearly demonstrated that the addition of TMZ to RT conferred a survival advantage for newly diagnosed GBM patients versus those receiving RT alone. Based on this trial, concurrent and adjuvant TMZ became the standard of care for the treatment of nGBM despite its added costs. A study by Mabasa and Taylor evaluated mOS and compared the cost effectiveness of TMZ and lomustine in patients with recurrent malignant glioma. ${ }^{96}$ A retrospective review of medical records from 41 patients was conducted. Patients receiving TMZ (mean 5.1 cycles) incurred a mean cost of \$Can10 746, while those receiving lomustine (mean 3.1 cycles) incurred a mean cost of \$Can129. Lomustine is a relatively inexpensive chemotherapeutic agent that is given orally once every 6 weeks. TMZ, a newer and more expensive agent, is given in a 5 consecutive day regimen every 28 days. This may explain the higher costs associated with TMZ treatment. In addition, patients with recurrent disease are generally resistant to chemotherapy. Since TMZ has not been shown to provide a survival benefit in recurrent disease versus lomustine, the use of the more expensive TMZ may not be cost effective in this patient population. The incremental cost-effectiveness ratio for each life-year gained with TMZ ranged from \$Can32 247 to \$Can162186. Since the study found no difference in mOS achieved with the two drugs, the authors concluded that lomustine was more cost effective than TMZ for the treatment of recurrent malignant glioma. ${ }^{96}$

Another study from Finland evaluated the cost effectiveness of TMZ versus PCV (procarbazine, lomustine, vincristine) treatment for rGBM patients. This study concluded that 1 extra life-month gained with TMZ cost $€ 2367,1$ extra progression-free life-month gained cost $€ 2165$, and the cost for each additional QALY gained was $€ 32471$ compared with PCV. The study also concluded that TMZ has a "high probability" of being cost effective compared with PCV for rGBM patients from a Finnish healthcare payer perspective.$^{97}$ Another small Finnish study concluded that the high acquisition costs of TMZ are compensated by prolonged home care and possibly by the ability to retain working capacity during TMZ treatment among patients with recurrent gliomas. ${ }^{98}$ Despite the additional costs associated with TMZ use, the improved survival and HRQoL in patients with malignant gliomas may justify the added costs to society.

\section{Patient group/population}

Certain populations may differentially benefit from TMZ treatment. For example, patients with tumors harboring methylated MGMT promoter were found to have better outcomes compared with those with tumors that had unmethylated MGMT promoters. ${ }^{99}$ The benefit of TMZ for the elderly (age $>70$ years) is unknown. ${ }^{28}$

\section{MGMT methylation status}

MGMT effectively reverses the cytotoxic action of TMZ and thus high MGMT expression levels are associated with TMZ resistance. Patients with silenced MGMT gene expression due to hypermethylation of MGMT promoters were shown to respond more favorably to concurrent TMZ/RT compared with those who had unmethylated MGMT promoters and high MGMT gene expression levels. ${ }^{99}$ In this study, tumor samples obtained from nGBM patients enrolled in the EORTC/ NCIC phase III study were analyzed for MGMT promoter methylation status by methylation-specific polymerase chain reaction techniques ${ }^{99}$ (Table 8 ).

\section{Elderly patients}

The treatment of elderly patients ( $\geq 70$ years old) with newly diagnosed malignant gliomas remains controversial. The pivotal EORTC/NCIC phase III trial evaluating concurrent and adjuvant TMZ for nGBM excluded patients of this age. ${ }^{28}$ One study has compared an abbreviated course of radiation (3 weeks) to the standard 6-week schedule in elderly patients diagnosed with malignant gliomas. The trial concluded that the 3-week schedule was better tolerated and achieved similar results compared with the 6-week regimen. ${ }^{100}$ Another phase III trial compared RT with standard supportive care in elderly patients with AA and GBM, and concluded that RT was associated with superior outcomes. ${ }^{101}$ Because of these findings, some neurooncologists advocate an abbreviated 3-week course of RT for elderly patients with 
nGBM and nAA. It is not clear whether the addition of TMZ concurrently and/or adjuvantly to this shortened RT treatment would provide any additional advantage to this elderly population. A trial sponsored by the NCIC/CTG is currently exploring the benefit of adding TMZ to RT in patients aged $>65$ years.

Another phase III trial assessed the efficacy of TMZ alone instead of RT in a group of 32 elderly patients. TMZ treatment resulted in a PR rate of $31 \%$, stable disease in a further $41 \%$ of patients, while $28 \%$ had evidence of progressive disease. This cohort of patients achieved a mOS of 6.2 months (13.3 months among those achieving a PR), which compares favorably with median survival associated with RT alone. ${ }^{102}$ In addition, TMZ treatment was associated with a relatively mild and often predictable side effect profile, which makes it an attractive treatment option for this frail patient population. ${ }^{102} \mathrm{~A}$ further retrospective study comparing TMZ alone $\left(150 \mathrm{mg} / \mathrm{m}^{2}\right.$ for 5 days every 28 days) with RT alone in 86 elderly patients with newly diagnosed malignant glioma reported a higher median survival among those treated with TMZ alone, but the difference did not reach statistical significance (6.0 versus 4.1 months). ${ }^{103}$

\section{Dosage, administration, and formulation}

TMZ is used for the treatment of newly diagnosed as well as recurrent GBM and AA. Based on the EORTC/NCIC trial, $\mathrm{TMZ}$ is given concurrently with RT at $75 \mathrm{mg} / \mathrm{m}^{2}$ followed by a minimum of six cycles of adjuvant TMZ. Adjuvant $\mathrm{TMZ}$ is administered at $150 \mathrm{mg} / \mathrm{m}^{2}$ for 5 days on cycle 1 and $200 \mathrm{mg} / \mathrm{m}^{2}$ from cycle 2 onward provided blood counts are adequate. The cycles are repeated every 28 days.

The management approach for patients with rAA is very similar to rGBM. ${ }^{104}$ Based on a trial by Yung et $\mathrm{al}^{54}$ demonstrating an ORR rate of $35 \%$ to TMZ, this agent is frequently employed for the treatment of rAA. ${ }^{54}$

Table 8 Effect of MGMT promoter methylation status on PFS and OS among patients receiving concurrent temozolomide/radiotherapy versus radiotherapy alone (adapted from Hegi et $\mathrm{a}^{99}$ )

\begin{tabular}{lll}
\hline Clinical endpoint & TMZ + RT $(\mathbf{n}=\mathbf{1 0 6})$ & $\mathbf{R T}(\mathbf{n}=\mathbf{1 0 0})$ \\
\hline Methylated MGMT (n) & 46 & 46 \\
PFS6m (\%) & 47.8 & 68.9 \\
2-year OS (\%) & 22.7 & 46.0 \\
Unmethylated MGMT (n) & 54 & 60 \\
PFS6m (\%) & 35.2 & 40.0 \\
2-year OS (\%) & $<2$ & 13.8 \\
\hline
\end{tabular}

Abbreviations:MGMT,O-6-methylguanine-DNA methyltransferase; OS, overall survival; PFS6m, progression-free survival at 6 months; RT, radiotherapy;TMZ, temozolomide.
When treating recurrent disease, TMZ is administered daily for 5 days similar to the adjuvant regimen mentioned above. On the other hand, nAA is typically treated with surgical resection followed by conformal RT with or without concurrent TMZ. The addition of concurrent TMZ is of no proven value in the treatment of nAA because of the lack of a RCT showing superiority of this approach over RT alone. ${ }^{105}$ An international collaborative trial that will answer this question is underway.

TMZ is available in capsules of 5, 20, 100, and $250 \mathrm{mg}$. It achieves approximately $100 \%$ bioavailability after oral administration. In addition, TMZ achieves excellent bloodbrain barrier penetration and the measured cerebrospinal fluid area under the curve (AUC) is typically $20 \%-40 \%$ of the plasma AUC. ${ }^{106}$

\section{Place in therapy}

Based on a number of trials, TMZ is quickly becoming a first-line agent for the treatment of malignant astrocytomas, both because of its proven efficacy as well as its safety profile. Concurrent TMZ and RT followed by adjuvant TMZ is currently the standard of care for nGBM. The role of TMZ for the management of nAA as well as for recurrent disease is less well defined at present but is widely used by neurooncologists for the first-line treatment of these disease entities.

In patients with rGBM, TMZ is associated with an increase of up to 6 months in PFS compared with PCB. Patients with rAA treated with a variety of chemotherapeutic agents achieved a PFS6m of $31 \%,{ }^{55}$ while rAA patients treated with TMZ in a phase II trial achieved a PFS6m of $46 \%$. The figures from these two trials are difficult to compare because of the differences in baseline patient characteristics. The worse outcome recorded in the trial conducted by Wong et $\mathrm{al}^{55}$ may be explained by the high proportion of patients with multiple recurrences and therefore with poorer prognoses.

Patients with rGBM and rAA had better HRQoL scores after treatment with TMZ. HRQoL was also not compromised in $\mathrm{nGBM}$ patients treated with concurrent $\mathrm{TMZ}$ and $\mathrm{RT}$ followed by adjuvant TMZ.

Concurrent TMZ with RT followed by adjuvant TMZ results in a 2.5-month prolongation of median survival among nGBM patients compared with patients receiving RT alone. More importantly, studies show that the probability of surviving to 2 years after diagnosis was only 1 in 10 for nGBM patients treated with RT alone, while patients treated with concurrent and adjuvant TMZ had a 1 in 4 chance of surviving to the 2-year time point. 
Newly-diagnosed patients with a methylated MGMT promoter as well as those in RPA class III (age $<50$ years and PS of 0 ) seem to benefit the most from the addition of TMZ. Further studies are clearly needed to further refine the dosing schedules as well as identify ideal agents to be used in combination with TMZ in order to improve outcomes for patients with malignant glioma.

\section{Acknowledgements}

Drs Omar and Mason have acted as consultants and advisors for, and received honoraria from, Schering-Plough Corporation.

\section{References}

1. CBTRUS (Central Brain Tumor Registry of the US). 2005-2006 Primary Brain Tumors in the United States Statistical Report, 1998-2002 Years Data Collected. Available at: http://www.cbtrus. org/reports//2005-2006/2006report.pdf.

2. Louis DN, von Deimling A. Hereditary tumor syndromes of the nervous system: overview and rare syndromes. Brain Pathol. 1995;5:145-151.

3. Konopka G, Bonni A. Signaling pathways regulating gliomagenesis. Curr Mol Med. 2003;3:73-84.

4. Tamura M, Misumi S, Kurosaki S, Shibasaki T, Ohye C. Anaplastic astrocytoma 14 years after radiotherapy for pituitary adenoma. No Shinkei Geka - Neurological Surgery. 1992;20:493-497 (in Japanese).

5. Tsang RW, Laperriere NJ, Simpson WJ, et al. Glioma arising after radiation therapy for pituitary adenoma. A report of four patients and estimation of risk. Cancer. 1993;72:2227-2233.

6. Neglia JP, Robison LL, Stovall M. New primary neoplasms of the central nervous system in survivors of childhood cancer: a report from the Childhood Cancer Survivor Study. J Natl Cancer Inst. 2006;98:1528-1537.

7. Hardell L, Mild KH, Carlberg M. Case-control study on the use of cellular and cordless phones and the risk for malignant brain tumours. Int J of Radiat Biol. 2002;78:931-936.

8. Muscat JE, Hinsvark M, Malkin M. Mobile telephones and rates of brain cancer. Neuroepidemiology. 2006;27:55-56.

9. Kim S, Dougherty ER, Shmulevich I, et al. Identification of combination gene sets for glioma classification. Mol Cancer The. 2002;1:1229-1236.

10. Ohgaki H, Dessen P, Jourde B, et al. Genetic pathways to glioblastoma: a population-based study. Cancer Res. 2004;64:6892-6899.

11. Ohgaki H, Kleihues P. Population-based studies on incidence, survival rates, and genetic alterations in astrocytic and oligodendroglial gliomas. J Neuropathol Exp Neurol. 2005;64:479-489.

12. Nozaki M, Tada M, Kobayashi H, et al. Roles of the functional loss of $\mathrm{p} 53$ and other genes in astrocytoma tumorigenesis and progression. Neuro Oncol. 1999;1:124-137.

13. Sarkar C, Ralte AM, Sharma MC, Mehta VS. Recurrent astrocytic tumours - a study of p53 immunoreactivity and malignant progression. Br J Neurosurg. 2002;16:335-342.

14. Wooten EC, Fults D, Duggirala R, et al. A study of loss of heterozygosity at 70 loci in anaplastic astrocytoma and glioblastoma multiforme with implications for tumor evolution. Neuro Oncol. 1999;1:169-176.

15. Kleihues R, Louis DN, Scheithauer BW, et al. The WHO classification of tumors of the nervous system. J Neuropathol Exp Neurol. 2002; 61:215-225; discussion 226-229.

16. Louis DN, Ohgaki H, Wiestler OD, et al. The 2007 WHO classification of tumours of the central nervous system. Acta Neuropathol. 2007;114:97-109.

17. O'Reilly SM, Newlands ES, Glaser MG, et al. Temozolomide: a new oral cytotoxic chemotherapeutic agent with promising activity against primary brain tumours. Eur J Cancer. 1993;29A:940-942.
18. Newlands ES, O'Reilly SM, Glaser MG, et al. The Charing Cross Hospital experience with temozolomide in patients with gliomas. Eur J Cancer. 1996;32A:2236-2241.

19. Weinstein MC, Stason WB. Foundations of cost-effectiveness analysis for health and medical practices. N Engl J Med. 1977;296: 716-721.

20. Weinstein MC. A QALY is a QALY-or is it? J Health Econ. 1988; 7:289-290.

21. Macdonald DR, Cascino TL, Schold SC Jr, Cairncross JG. Response criteria for phase II studies of supratentorial malignant glioma. J Clin Oncol. 1990;8:1277-1280.

22. Aaronson NK, Ahmedzai S, Bergman B, et al. The European Organization for Research and Treatment of Cancer QLQ-C30: a quality-of-life instrument for use in international clinical trials in oncology. J Nat Can Inst. 1993;85:365-376.

23. Osoba D, Aaronson NK, Muller M, et al. The development and psychometric validation of a brain cancer quality-of-life questionnaire for use in combination with general cancer-specific questionnaires. Qual Life Res. 1996;5:139-150.

24. King MT. The interpretation of scores from the EORTC quality of life questionnaire QLQ-C30. Qual Life Res. 1996;5:555-567.

25. Osoba D, Rodrigues G, Myles J, Zee B, Pater J. Interpreting the significance of changes in health-related quality-of-life scores. J Clin Oncol. 1998;16:139-144.

26. Yung WK, Albright RE, Olson J, et al. A phase II study of temozolomide vs procarbazine in patients with glioblastoma multiforme at first relapse. Br J Cancer. 2000;83:588-593.

27. Stupp R, Hegi ME, van den Bent MJ, et al. Changing paradigms - an update on the multidisciplinary management of malignant glioma. Oncologist. 2006;11:165-180.

28. Stupp R, Mason WP, van den Bent MJ. Radiotherapy plus concomitant and adjuvant temozolomide for glioblastoma. $N \mathrm{Engl} \mathrm{J} \mathrm{Med.}$ 2005;352:987-996.

29. Simpson L, Galanis E. Recurrent glioblastoma multiforme: advances in treatment and promising drug candidates. Expert Rev Anticancer Ther. 2006;6:1593-1607.

30. Franceschi E, Cavallo G, Scopece L, et al. Phase II trial of carboplatin and etoposide for patients with recurrent high-grade glioma. $\mathrm{Br} J$ Cancer. 2004;91:1038-1044.

31. Rao RD, Krishnan S, Fitch TR, et al. Phase II trial of carmustine, cisplatin, and oral etoposide chemotherapy before radiotherapy for grade 3 astrocytoma (anaplastic astrocytoma): results of North Central Cancer Treatment Group trial 98-72-51.Int J Radiat Oncol Biol Phys. 2005;61:380-386

32. Chamberlain MC, Tsao-Wei DD, Groshen S. Salvage chemotherapy with cyclophosphamide for recurrent temozolomide-refractory anaplastic astrocytoma. Cancer. 2006;106:172-179.

33. Guyotat J, Signorelli F, Frappaz D, Madarassy G, Ricci AC, Bret P. Is reoperation for recurrence of glioblastoma justified? Oncol Rep. 2000;7:899-904.

34. Bauman GS, Sneed PK, Wara WM, et al. Reirradiation of primary CNS tumors. Int J Radiat Oncol Biol Phys. 1996;36:433-441.

35. Veninga T, Langendijk HA, Slotman BJ, et al. Reirradiation of primary brain tumours: survival, clinical response and prognostic factors Radiother Oncol. 2001;59:127-137.

36. Voynov G, Kaufman S, Hong T, Pinkerton A, Simon R, Dowsett R. Treatment of recurrent malignant gliomas with stereotactic intensity modulated radiation therapy. Am J Clin Oncol. 2002;25:606-611.

37. Walker MD, Green SB, Byar DP, et al. Randomized comparisons of radiotherapy and nitrosoureas for the treatment of malignant glioma after surgery. N Engl J Med. 1980;303:1323-1329.

38. Chang CH, Horton J, Schoenfeld D, et al. Comparison of postoperative radiotherapy and combined postoperative radiotherapy and chemotherapy in the multidisciplinary management of malignant gliomas. A joint Radiation Therapy Oncology Group and Eastern Cooperative Oncology Group study. Cancer. 1983;52: 997-1007. 
39. Shapiro WR, Green SB, Burger PC, Mahaley MS Jr, et al. Randomized trial of three chemotherapy regimens and two radiotherapy regimens in postoperative treatment of malignant glioma. Brain Tumor Cooperative Group Trial 8001. J Neurosurg. 1989;71:1-9.

40. MRCBTWP (Medical Research Council Brain Tumor Working Party). Randomized trial of procarbazine, lomustine, and vincristine in the adjuvant treatment of high-grade astrocytoma: a Medical Research Council trial. J Clin Oncol. 2001;19:509-518.

41. Stewart LA. Chemotherapy in adult high-grade glioma: a systematic review and meta-analysis of individual patient data from 12 randomised trials. Lancet. 2002;359:1011-1018.

42. Denny BJ, Wheelhouse RT, Stevens MF, Tsang LL, Slack JA. NMR and molecular modeling investigation of the mechanism of activation of the antitumor drug temozolomide and its interaction with DNA. Biochemistry. 1994;33:9045-9051.

43. Pearl LH, Savva R. DNA repair in three dimensions. Trends Biochem Sci. $1995 ; 20: 421-426$.

44. Hotta T, Saito Y, Fujita H, et al. O6-alkylguanine-DNA alkyltransferase activity of human malignant glioma and its clinical implications. J Neurooncol. 1994;21:135-140.

45. Gerson SL. MGMT: its role in cancer aetiology and cancer therapeutics. Nature Reviews. Cancer. 2004;4:296-307.

46. NCI-CTC (National Cancer Institute Common Toxicity Criteria). Cancer Therapy Evaluation Program, Common Terminology Criteria for Adverse Events, (DCTD, NCI, NIH, DHHS). 2003. http://ctep. cancer.gov/forms/CTCAEv3.pdf.

47. Ganière V, Christen G, Bally F, et al. Listeria brain abscess, Pneumocystis pneumonia and Kaposi's sarcoma after temozolomide. Nat Clin Pract Oncol. 2006;3:339-343.

48. Maldonado F, Limper AH, Lim KG, Aubrey MC. Temozolomideassociated organizing pneumonitis. Mayo Clin Proc. 2007;82: 771-773.

49. Brada M, Ashley S, Dowe A, et al. Neoadjuvant phase II multicentre study of new agents in patients with malignant glioma after minimal surgery. Report of a cohort of 187 patients treated with temozolomide. Ann Oncol. 2005;16:942-949.

50. Baumann F, Bjeljac M, Kollias SS, Baumert BG, et al. Combined thalidomide and temozolomide treatment in patients with glioblastoma multiforme. J Neurooncol. 2004;67:191-200.

51. Newlands ES, Foster T, Zaknoen S. Phase I study of temozolamide (TMZ) combined with procarbazine (PCB) in patients with gliomas. Br J Cancer. 2003;89:248-251.

52. Chang SM, Prados MD, Yung WK, et al. Phase II study of neoadjuvant 1 , 3-bis (2-chloroethyl)-1-nitrosourea and temozolomide for newly diagnosed anaplastic glioma: a North American Brain Tumor Consortium Trial. Cancer. 2004;100:1712-1706.

53. Brada M, Hoang-Xuan K, Rampling R. Multicenter phase II trial of temozolomide in patients with glioblastoma multiforme at first relapse. Ann Oncol. 2001;12:259-266.

54. Yung WK, Prados MD, Yaya-Tur R, et al. Multicenter phase II trial of temozolomide in patients with anaplastic astrocytoma or anaplastic oligoastrocytoma at first relapse. Temodal Brain Tumor Group. J Clin Oncol. 1999;17:2762-2771.

55. Wong ET, Hess KR, Gleason MJ, et al. Outcomes and prognostic factors in recurrent glioma patients enrolled onto phase II clinical trials. J Clin Oncol. 1999;17:2572-2578.

56. Herrlinger U, Rieger J, Koch D, et al. Phase II trial of lomustine plus temozolomide chemotherapy in addition to radiotherapy in newly diagnosed glioblastoma: UKT-03. J Clin Oncol. 2006; 24:4 412-417.

57. Wedge SR, Porteous JK, Glaser MG, Marcus K, Newlands ES. In vitro evaluation of temozolomide combined with X-irradiation. Anticancer Drugs. 1997;8:92-97.

58. van Rijn J, Heimans JJ, van den Berg J, van der Valk R Slotman BJ. Survival of human glioma cells treated with various combination of temozolomide and X-rays. Int J Radiat Oncol Bio Phys. 2000;47: 779-784.
59. Stupp R, Dietrich PY, Ostermann Kraljevic S, et al. Promising survival for patients with newly diagnosed glioblastoma multiforme treated with concomitant radiation plus temozolomide followed by adjuvant temozolomide. J Clin Oncol. 2002;20:1375-1382.

60. Athanassiou H, Synodinou M, Maragoudakis E, et al. Randomized phase II study of temozolomide and radiotherapy compared with radiotherapy alone in newly diagnosed glioblastoma multiforme. $J$ Clin Oncol. 2005:23:2372-2377.

61. Hosli P, Sappino AP, de Tribolet N, Dietrich, PY. Malignant glioma: should chemotherapy be overthrown by experimental treatments? Ann Oncol. 1998;9:589-600.

62. Pech IV, Peterson K, Cairncross JG. Chemotherapy for brain tumors. Oncology (Williston Park). 1998;12:537-543.

63. Groves MD, Puduvalli VK, Chang SM, et al. A North American brain tumor consortium (NABTC 99-04) phase II trial of temozolomide plus thalidomide for recurrent glioblastoma multiforme. J Neurooncol. 2007;81:271-277.

64. D'Atri S, Graziani G, Lacal PM, et al. Attenuation of O(6)-methylguanineDNA methyltransferase activity and mRNA levels by cisplatin and temozolomide in jurkat cells. J Pharmacol Exp Ther. 2000; 294:664-671.

65. Brandes AA, Basso U, Reni M, et al. First-line chemotherapy with cisplatin plus fractionated temozolomide in recurrent glioblastoma multiforme: a phase II study of the Gruppo Italiano Cooperativo di Neuro-Oncologia. J Clin Oncol. 2004;22:1598-1604.

66. Silvani A, Eoli M, Salmaggi A, et al. Phase II trial of cisplatin plus temozolomide, in recurrent and progressive malignant glioma patients. J Neurooncol. 2004;66:203-208.

67. Awada A, Gil T, Sales F, et al. Prolonged schedule of temozolomide (Temodal) plus liposomal doxorubicin (Caelyx) in advanced solid cancers. Anticancer Drugs. 2004;15:499-502.

68. Chua SL, Rosenthal MA, Wong SS, et al. Phase 2 study of temozolomide and Caelyx in patients with recurrent glioblastoma multiforme. Neurooncol. 2004;6:38-43.

69. Tonn JC, Kerkau S, Hanke A, et al. Effect of synthetic matrixmetalloproteinase inhibitors on invasive capacity and proliferation of human malignant gliomas in vitro. IntJ Cancer. 1999;80:764-772.

70. Groves MD, Puduvalli VK, Hess KR, et al. Phase II trial of temozolomide plus the matrix metalloproteinase inhibitor, marimastat, in recurrent and progressive glioblastoma multiforme. J Clin Oncol. 2002;20:1383-1388.

71. Brandes AA, Ermani M, Basso U, et al. Temozolomide in patients with glioblastoma at second relapse after first line nitrosourea-procarbazine failure: a phase II study. Oncology. 2002;63:38-41.

72. Tolcher AW, Gerson SL, Denis L, et al. Marked inactivation of O6-alkylguanine-DNA alkyltransferase activity with protracted temozolomide schedules. Br J Cancer. 2003;88:1004-2011.

73. Khan RB, Raizer JJ, Malkin MG, Bazylewicz KA, Abrey LE. A phase II study of extended low-dose temozolomide in recurrent malignant gliomas. Neurooncology. 2002;4:39-43.

74. Fountzilas G, Karkavelas G, Kalogera-Fountzila A, et al. Post-operative combined radiation and chemotherapy with temozolomide and irinotecan in patients with high-grade astrocytic tumors. A phase II study with biomarker evaluation. Anticancer Res. 2006;26:4675-4686.

75. Osoba D, Brada M, Yung WK, Prados M. Health-related quality of life in patients treated with temozolomide versus procarbazine for recurrent glioblastoma multiforme. J Clin Oncol. 2000a;18: 1481-1491.

76. Osoba, D, Brada M, Yung WK, Prados, MD. Health-related quality of life in patients with anaplastic astrocytoma during treatment with temozolomide. Eur J Cancer. 2000b;36:1788-1795.

77. Hamilton DA. Adding concomitant and adjuvant temozolomide to radiotherapy does not reduce health-related quality of life in people with glioblastoma. Cancer Treat Rev. 2006;32:483-486.

78. Newlands ES, Blackledge GR, Slack JA, et al. Phase I trial of temozolomide (CCRG 81045: M\&B 39831: NSC 362856). Br J Cancer. 1992;65:287-291. 
79. Carson KA, Grossman SA, Fisher JD, Shaw EG. Prognostic factors for survival in adult patients with recurrent glioma enrolled onto the new approaches to brain tumor therapy CNS consortium phase I and II clinical trials. J Clin Oncol. 2007;25:2601-2606.

80. Compostella A, Tosoni A, Blatt V, Franceschi E, Brandes AA. Prognostic factors for anaplastic astrocytomas. J Neurooncol. 2007;81:295-303.

81. Devaux BC, O'Fallon JR, Kelly PJ. Resection, biopsy, and survival in malignant glial neoplasms. A retrospective study of clinical parameters, therapy, and outcome. J Neurosurg. A993;78:767-775.

82. Laws ER, Parney IF, Huang W, et al. Survival following surgery and prognostic factors for recently diagnosed malignant glioma: data from the Glioma Outcomes Project. J Neurosurg. 2003;99:467-473.

83. Lacroix M, Abi-Said D, Fourney DR, et al. A multivariate analysis of 416 patients with glioblastoma multiforme: prognosis, extent of resection, and survival. J Neurosurg. 2001;95:190-198.

84. Vuorinen V, Hinkka S, Farkkila M, Jaaskelainen J. Debulking or biopsy of malignant glioma in elderly people - a randomised study. Acta Neurochir (Wien). 2003;145:5-10.

85. Levivier M, Violon P, Brotchi J, Goldman S, Kowalczuk A. Quantitative imaging study of extent of surgical resection and prognosis of malignant astrocytomas. Neurosurgery. 1998;43:398-399.

86. Curran WJ Jr, Scott CB, Horton J, et al. Recursive partitioning analysis of prognostic factors in three Radiation Therapy Oncology Group malignant glioma trials. J Natl Cancer Inst. 1993;85:704-710.

87. Mirimanoff RO, Gorlia T, Mason W, et al. Radiotherapy and temozolomide for newly diagnosed glioblastoma: recursive partitioning analysis of the EORTC 26981/22981-NCIC CE3 phase III randomized trial. J Clin Oncol. 2006;24:2563-2569.

88. NCIC (National Cancer Institute of Canda). Temozolomide and Everolimus in Treating Patients With Newly Diagnosed, Recurrent, or Progressive Malignant Glioblastoma Multiforme (Study Sponsor: National Cancer Institute of Canada). 2007. Available at: http:// clinicaltrials.gov/ct2/show/NCT00387400.

89. Schering Canada Inc. The Effects of Continuous 28-Day (28/28) Temozolomide Chemotherapy in Subjects With Recurrent Malignant Glioma Who Have Failed the Conventional 5-Day (5/28) Treatment (P04601AM1). 2007. Available at: http://clinicaltrials.gov/ct2/show/NCT00392171.

90. Quinn JA, Desjardins A, Weingart J, et al. Phase I trial of temozolomide plus O6-benzylguanine for patients with recurrent or progressive malignant glioma. J Clin Oncol. 2005;23:7178-7187.

91. Koch D, Hundsberger T, Boor S, Kaina B. Local intracerebral administration of $\mathrm{O}(6)$-benzylguanine combined with systemic chemotherapy with temozolomide of a patient suffering from a recurrent glioblastoma. J Neurooncol. 2007;82:85-89.

92. Wasserfallen JB, Ostermann S, Pica A, et al. Can we afford to add chemotherapy to radiotherapy for glioblastoma multiforme? Cost-identification analysis of concomitant and adjuvant treatment with temozolomide until patient death. Cancer. 2004;101:2098-2105.
93. Prosser LA, Stinnett AA, Goldman PA, et al. Cost-effectiveness of cholesterol-lowering therapies according to selected patient characteristics. Ann Intern Med. 2000;132:769-779.

94. Wasserfallen JB, Ostermann S, Leyvraz S, Stupp R. Cost of temozolomide therapy and global care for recurrent malignant gliomas followed until death. Neurooncology. 2005;7:189-195.

95. Dinnes J, Cave C, Huang S, Major K, Milne R. The effectiveness and cost-effectiveness of temozolomide for the treatment of recurrent malignant glioma: a rapid and systematic review. Health Technol Assess. 2001;5:1-73.

96. Mabasa VH, Taylor SC. Re-evaluation of the cost effectiveness of temozolomide for malignant gliomas in British Columbia. J Oncol Pharm Pract. 2006;12:105-111.

97. Martikainen JA, Kivioja A, Hallinen T, Vihinen P. Economic evaluation of temozolomide in the treatment of recurrent glioblastoma multiforme. Pharmacoeconomics. 2005;23:803-805.

98. Maenpaa HO, Aaltonen K, Mantyla R, Minn, H. Temozolomide treatment in glioma - experiences in two university hospitals in Finland. Acta Oncol. 2004;43:579-584.

99. Hegi ME, Diserens AC, Gorlia T. MGMT gene silencing and benefit from temozolomide in glioblastoma. $N$ Eng $l \mathrm{~J} \mathrm{Med.} \mathrm{2005;352:}$ 997-1003.

100. Roa W, Brasher PM, Bauman G. Abbreviated course of radiation therapy in older patients with glioblastoma multiforme: a prospective randomized clinical trial. J Clin Oncol. 2004;22:1583-1588.

101. Keime-Guibert F, Chinot O, Taillandier L. Radiotherapy for glioblastoma in the elderly. N Engl J Med. 2007;356:1527-1535.

102. Chinot OL, Barrie M, Frauger E, et al. Phase II study of temozolomide without radiotherapy in newly diagnosed glioblastoma multiforme in an elderly population. Cancer. 2004;100:2208-2214.

103. Chang S, Theodosopoulos R Sneed P. Multidisciplinary management of adult anaplastic astrocytomas. Semin Radiat Oncol. 2001;11: $163-169$.

104. Glantz M, Chamberlain M, Liu Q, Litofsky NS, Recht LD. Temozolomide as an alternative to irradiation for elderly patients with newly diagnosed malignant gliomas. Cancer. 2003;97: 2262-2266.

105. Stupp R, Reni M, Gatta G, Mazza E, Vecht C. Anaplastic astrocytoma in adults. Crit Rev Oncol Hematol. 2007;63:72-80.

106. Ostermann S, Csajka C, Buclin T, et al. Plasma and cerebrospinal fluid population pharmacokinetics of temozolomide in malignant glioma patients. Clin Cancer Res. 2004;10:3728-3736.

107. Athanassiou AE, Potamianou A, Bountouroglou N, et al. Methotrexate, etoposide, ifosamide and cisplantin (MVIP): an effective first-line therapy for IGCCC intermediate/poor prognosis patients with germ-cell tumors. Single institution experience. $J B U O N$. $2002 ; 7: 35-41$.
Core Evidence

\section{Publish your work in this journal}

Core Evidence is an international, peer-reviewed open-access journal evaluating the evidence underlying the potential place in therapy of drugs throughout their development lifecycle from preclinical to postlaunch. The focus of each review is to evaluate the case for a new drug or class in outcome terms in specific indications and patient

\section{Dovepress}

groups. The manuscript management system is completely online and includes a very quick and fair peer-review system, which is all easy to use. Visit http://www.dovepress.com/testimonials.php to read real quotes from published authors. 